\title{
A numerical study of steady and unsteady viscoelastic flow past bounded cylinders
}

\author{
Paulo J. Oliveira ${ }^{\mathrm{a}, *}$, Amílcar I.P. Miranda ${ }^{\mathrm{b}}$ \\ ${ }^{a}$ Departamento de Engenharia Electromecânica, Unidade Materiais Têxteis e Papeleiros, Universidade da Beira Interior, 6201-001 Covilhã, Portugal \\ ${ }^{\mathrm{b}}$ Departamento de Matemática, Universidade da Beira Interior, 6201-001 Covilhã, Portugal
}

Received 20 October 2004; received in revised form 11 January 2005; accepted 12 February 2005

\begin{abstract}
We consider two-dimensional, inertia-free, flow of a constant-viscosity viscoelastic fluid obeying the FENE-CR equation past a cylinder placed symmetrically in a channel, with a blockage ratio of 0.5 . Through numerical simulations we show that the flow becomes unsteady when the Deborah number (using the usual definition) is greater than $D e \approx 1.3$, for an extensibility parameter of the model of $L^{2}=144$. The transition from steady to unsteady flow is characterised by a small pulsating recirculation zone of size approximately equal to 0.15 cylinder radius attached to the downstream face of the cylinder. There is also a rise in drag coefficient, which shows a sinusoidal variation with time. The results suggest a possible triggering mechanism leading to the steady three-dimensional Gortler-type vortical structures, which have been observed in experiments of the flow of a viscoelastic fluid around cylinders. The results reveal that the reason for failure of the search for steady numerical solutions at relatively high Deborah numbers is that the two-dimensional flow separates and eventually becomes unsteady. For a lower extensibility parameter, $L^{2}=100$, a similar recirculation is formed given rise to a small standing eddy behind the cylinder which becomes unsteady and pulsates in time for Deborah numbers larger than $D e \approx 4.0-4.5$.
\end{abstract}

(C) 2005 Elsevier B.V. All rights reserved.

Keywords: Cylinder; Viscoelastic; FENE-CR; Unsteady flow; Elastic instability; Flow separation at $R e=0$

\section{Introduction}

Rather than being resolved by improvements in numerical procedures and the increase in computer power, with the passage of time new challenges in computational rheology seem to have arisen. Early transition to three-dimensional steady flow and/or time dependency seem to be the rule rather than the exception for nominally two-dimensional, steady flow. For relatively strong inertial flows in the laminar regime, viscoelasticity has been found to promote stability in the sense of increasing the critical Reynolds number. Examples include the pitchfork transition of two-dimensional steady symmetric to two-dimensional steady asymmetric states in flows through expansions (Oliveira [1]) and the decreasing frequency of the unsteady instability of shear layers (vortex shedding, e.g. Oliveira [2], Sahin and Owens [3]). However,

\footnotetext{
* Corresponding author. Fax: +351 275329972.

E-mail address: pjpo@ubi.pt (P.J. Oliveira).
}

for low or negligible inertia, the situation is reversed. In the low Reynolds-number range, elastic effects predominate and nonlinear phenomena related to the constitutive equations of the non-Newtonian media are common (McKinley [4]; McKinley et al. [5]). It is becoming more and more frequent to see studies where the so-called benchmark flows used in computational rheology are found to reveal ever-more complex behaviour. For example, "steady" flow through contractions is seen to present unsteady characteristics, with pulsating formation and detachment of "lip vortices" (e.g. Oliveira [6]). While such observations were already common in experimental work (see, for example, the photographic compilation of Boger and Walters [7] and the excellent review of the subject, encompassing both flows around cylinders and in contractions, by McKinley [4]), their resolution and capture eluded numerical simulation for many years. Even simpler flows, such as the rectilinear channel flow of a viscoelastic fluid, such as the simplified PTT fluid, do in fact give rise to some form of instability, as shown by Grillet et al. [8]. 


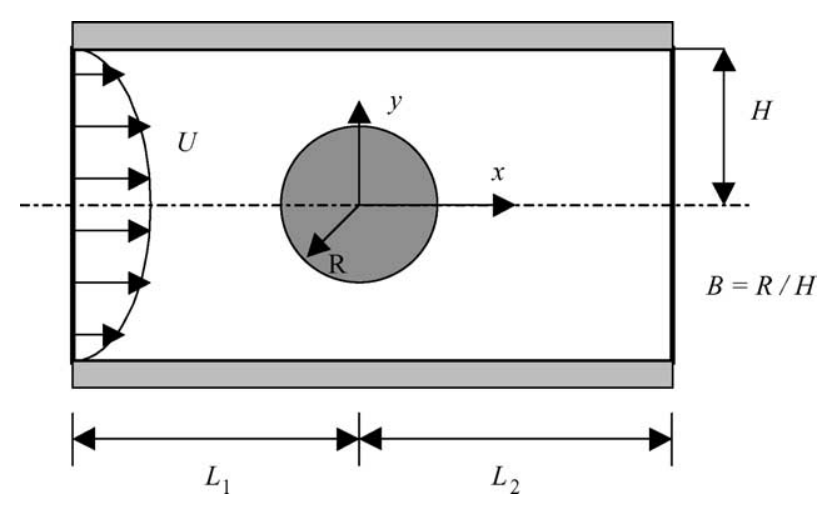

Fig. 1. Sketch of the flow geometry $\left(L_{1}=20 R ; L_{2}=60 R\right)$.

The topic of the present study is the flow past cylinders, mainly under the situation in which a single cylinder is placed symmetrically in a plane channel. This configuration (Fig. 1) has been used as a numerical benchmark problem since the proposal by Brown and McKinley [9] and a number of works dealing with it have emerged [10-20]. Most of these have considered either the upper convected Maxwell (UCM) or the Oldroyd-B models (Refs. [11-16,18,20]), and the main quantity of interest, representing the overall flow feature around the cylinder under creeping flow conditions $(R e=0)$, has been the evolution of the drag coefficient $C_{\mathrm{D}}$ with the Deborah number $D e$. Noteworthy were the numerical results of Fan et al. [11] and of Alves et al. [15] which, in both instances, revealed almost coincident $C_{\mathrm{D}}$ variations up to a Deborah number of $D e \approx 1$ based on calculations using different numerical methodologies. The former group used a highly accurate $h-p$ finite element formulation while the second group employed the finite volume method (FVM) on extremely refined meshes with second-order high-resolution schemes. While it has been recognised that accurate predictions of $C_{\mathrm{D}}$ does not necessarily mean accurate predictions of the detailed flow structure (see, e.g. $[11,18]$ ), particularly so far as the flow features along the downstream cylinder wake are concerned, it is worth noting that many predictions of $C_{\mathrm{D}}$ deviate at relatively low levels of elasticity (say $D e \approx 0.5$ ) from the results of these two studies, a situation that may be considered as an indication of inaccurate predictions.

A major area of concern is the level of De attained in the previous predictions with the UCM or the Oldroyd-B models (typically $D e \leq \sim 1$ ). The question which arises is "are there physical reasons for those limitations, or do they reflect a numerical limitation related to the inability of present-day numerical procedures for non-Newtonian flow simulations?" The flow around the cylinder is devoid of geometric singular points, in the sense of features like re-entrant corners typical of contraction flows, and that has been one of the reasons for the preference of the former type of flows. Lack of geometric singularities then calls for a justification for the numerical failure based on possible constitutive singularities. It is well known that both the UCM and the Oldroyd-B models have singularities in the extensional viscosities for a finite, rela- tively low, extensional rate. On the other hand, those models are known $[4,21]$ not to represent adequately, except at very low shear rates, the rheometrical behaviour of Boger fluids (i.e. approximately constant viscosity, high solvent viscosity, polymer solutions) for which they were supposed to be well suited. In this respect, models based on the notion of finite extensibility are more accurate in representing the viscometric functions of some Boger fluids [4], even when the viscosity itself is still considered to be constant with shear rate. It is for this reason that we have decided to use the FENE-CR model [22] described in Section 2.

Although numerical simulations of flow past a cylinder avoid the inevitable problems associated with the existence of geometrical singularities, for example in the case of flow through contractions, they still present a number of challenges: thin viscous boundary layers which develop on the cylinder surface; co-existence of zones having predominantly shear-flow characteristics with zones of predominantly extensional characteristics; formation of a thin viscous wake behind the cylinder (bi-refringence strand).

In past work with our FVM in this geometry [15], only quasi-linear constitutive models have been considered, namely the upper-convected Maxwell and the Oldroyd-B models. As mentioned above, it was possible to pursue the computations up to Deborah numbers $(D e)$ of the order of unity and still obtain steady-state solutions. For higher levels of elasticity, as measured by a Deborah number defined in the usual way in terms of the average velocity in the channel and the cylinder radius, steady solutions could not be obtained. In the present work, we consider a rheological model with finite extensibility (measured by parameter $L^{2}$ ), the modified FENE-CR model [23], so that numerical problems associated with infinite extensional viscosities can be circumvented and higher levels of elasticity can (in principle) be reached. For the symmetrical geometry, we present accurate results for the drag coefficient for Deborah numbers up to 10. When the full cylinder is considered, without calling for symmetry about the channel centre plane, we show that the flow becomes unsteady and periodic at a Deborah number of about 1.3 (for $L^{2}=144$ ), with a small time-periodic separation bubble behind the cylinder.

\section{Governing equations}

As noted in Section 1, many of the earlier works dealing with viscoelastic flow around a cylinder (Liu et al. [10], Fan et al. [11], Sun et al. [12], Dou and Phan-Thien [13], Alves et al. [15]) employed either the UCM or the Oldroyd-B models to represent the fluid rheology. In the case of the latter model, it has been common to choose the solvent viscosity ratio as $\beta=\eta_{\mathrm{s}} / \eta_{0}=0.59$, where $\eta_{0}$ is the zero-shear-rate viscosity given as the sum of solvent and "polymeric" contributions, $\eta_{0}=\eta_{\mathrm{s}}+\eta_{p}$. That particular value was based on the measured data for the MIT Boger fluid (McKinley et al. [24]), a solution of polyisobutylene dissolved in a polybutene and 
tetradecane mixture. Accurate numerical solutions based on those models were given by $[11,15]$, which fully agreed with each other and where a maximum Deborah number value of around $D e \approx 1$ was found for steady-state numerical solutions. If attention is still focused on dilute or semi-dilute polymer solutions, the next step in terms of complexity of molecular-based rheological modeling is to introduce finite dumbbell extensibility, and thus evolve from the Oldroyd-B to the FENE-type models (see Bird et al. [25]). Some authors have already followed that route (see $[24,10,17,19])$. The expectation here is that by introducing more realism into the physical representation of the polymer molecular behaviour, at the same time the numerical solution difficulties will be softened and higher values of Deborah number should be attainable. Since we also want to separate elastic effects from those due to shear thinning, we consider first the FENE-type equation proposed by Chilcott and Rallison [22], which may be written in terms of a configuration tensor $(\boldsymbol{A})$ as:

$$
\lambda \stackrel{\nabla}{\boldsymbol{A}}=-f[A](\boldsymbol{A}-\boldsymbol{I})
$$

from which the extra stress tensor can be expressed explicitly by means of a Kramers expression:

$$
\boldsymbol{\tau}=\frac{\eta_{p} f[A]}{\lambda}(\boldsymbol{A}-\boldsymbol{I})
$$

where $\lambda$ is the relaxation time, $\boldsymbol{I}$ the identity tensor, the square brackets indicate a functional dependency, and $(\cdot)^{\nabla}$ denotes the upper convected derivative. Eqs. (1) and (2) comprise the so-called FENE-CR model which, although derived from empirical considerations (see discussion in [26], we note that the empiricisms are of the same level as those invoked for the well-known Peterlin approximation in the FENE-P model; see below), tend to provide a much better representation of the material functions of a Boger fluid, especially regarding the shear-thinning of the normal-stress coefficient. The stretch function $f[A]$ in Eq. (1) depends on the extensibility parameter $L^{2}$, which represents the ratio of the maximum to equilibrium average dumbbell extensions, and is given by:

$$
f[A]=\frac{1}{1-\operatorname{Tr}(\boldsymbol{A}) / L^{2}}
$$

This is the same function of the FENE-P model (referred to as $Z$ in the original paper, Bird et al. [27]) and is derived by invoking Peterlin's approximation $(\langle f[Q \boldsymbol{Q}]\rangle \approx f[\langle\boldsymbol{Q Q}\rangle]$ where $\boldsymbol{A}=\langle\boldsymbol{Q} \boldsymbol{Q}\rangle, \boldsymbol{Q}$ is the dumbbell end-to-end vector and $\langle\cdot\rangle$ a statistical average).

For a matter of computational convenience, and indeed to spare memory resources, it may be advantageous to substitute the stress tensor for the conformation tensor in Eqs. (1) and (2), giving:

$\boldsymbol{\tau}+\lambda \frac{\mathrm{D}}{\mathrm{D} t}\left(\frac{\boldsymbol{\tau}}{f}\right)=\eta_{p}\left(\nabla \boldsymbol{u}+\nabla \boldsymbol{u}^{\mathrm{T}}\right)+\frac{\lambda}{f}\left(\boldsymbol{\tau} \cdot \nabla \boldsymbol{u}+\nabla \boldsymbol{u}^{\mathrm{T}} \cdot \boldsymbol{\tau}\right)$ and

$f[\tau]=\frac{L^{2}+\left(\lambda / \eta_{p}\right) \operatorname{Tr}(\boldsymbol{\tau})}{L^{2}-3}$

Eq. (4) represents the stress tensor formulation of the FENE-CR constitutive equation, to be solved in conjunction with the equation of motion:

$\rho \frac{\mathrm{D} \boldsymbol{u}}{\mathrm{D} t}=-\nabla p+\eta_{\mathrm{s}} \boldsymbol{\nabla} \cdot\left(\boldsymbol{\nabla u}+\nabla \boldsymbol{u}^{\mathrm{T}}\right)+\nabla \cdot \tau$

and the incompressibility constraint:

$\nabla \cdot \boldsymbol{u}=0$

In these equations, $p$ is the pressure, $\rho$ the fluid density, and the solvent stress was assumed to follow a Newtonian relationship. An additional simplification which allows existing numerical methods to be used for solving Eq. (4) with only minor modifications is to discard the variation of $\mathrm{D}(1 / f) / \mathrm{D} t$ and write:

$\boldsymbol{\tau}+\frac{\lambda}{f} \frac{\mathrm{D}}{\mathrm{D} t}(\boldsymbol{\tau})=\eta_{p}\left(\nabla \boldsymbol{u}+\nabla \boldsymbol{u}^{\mathrm{T}}\right)+\frac{\lambda}{f}\left(\boldsymbol{\tau} \cdot \nabla \boldsymbol{u}+\nabla \boldsymbol{u}^{\mathrm{T}} \cdot \boldsymbol{\tau}\right)$

This represents a modified FENE-CR model (designated by FENE-MCR) first used by Coates et al. [23] in 1992 and later by a number of other authors in a range of studies. It should be emphasised that the steady-state rheometrical functions of FENE-CR and FENE-MCR are identical so that only minor differences are anticipated in steady-state complex flows. The reason is that the effect of $\boldsymbol{u} \cdot \nabla(1 / f)$ can be important only in strong convective regions in a flow. This argument will be demonstrated with the present numerical results for the flow around a cylinder. It is also important to note at this point that a number of other authors $([24,10,3])$ have simulated the flow of the FENE-MCR fluid around a confined cylinder and in this sense precise benchmark solutions, for exactly the same fluid model are needed, such as we present here.

\section{Discretisation and solution procedure}

A fully implicit, sequential (decoupled) algorithm is employed to solve the set of differential governing equations given in the previous section, which must first be transformed into algebraic equations by means of a finite volume discretisation on a collocated, non-orthogonal mesh. Both the spatial discretisation, which employs the CUBISTA scheme of Alves et al. [28], and the temporal discretisation, with a three time level representation of the unsteady terms in the equations, are formally second-order accurate. The algorithm was explained in detail in Oliveira [2] and only an outline is given here.

The discretised constitutive equation for any cell $P$ is:

$$
\begin{aligned}
a_{P}^{\tau} \boldsymbol{\tau}_{P}^{(n+1)}= & \sum_{F} a_{F}^{\tau} \boldsymbol{\tau}_{F}^{(n+1)}+\left\{S_{\tau}\left[\nabla \boldsymbol{u}^{*}\right]+S_{\tau}^{\mathrm{HOS}}\right. \\
& \left.+\frac{\lambda_{\mathrm{ef}} V}{\delta t}\left(2.0 \boldsymbol{\tau}_{P}^{(n)}-0.5 \boldsymbol{\tau}_{P}^{(n-1)}\right)\right\}
\end{aligned}
$$


with $a_{P}^{\tau}=\left(1.5 \frac{\lambda_{\mathrm{ef}} V}{\delta t}+V+\sum_{F} a_{F}^{\tau}\right) ; \quad \lambda_{\mathrm{ef}}=\lambda / f\left[\tau_{P}^{*}\right]$, and where the stress coefficients $a_{F}^{\tau}$ are made up of convective fluxes. These are evaluated at the cell faces located between cell $P$ and any of its neighbouring cells. The stress source terms $S_{\tau}$ comprise a part of the upper-convected derivative proportional to velocity gradients $\boldsymbol{\nabla} \boldsymbol{u}$ (explicitly indicated in Eq. (9) in order to highlight the interrelation between the kinematics and the stress fields), and a deferred correction part related to the implementation of the high-resolution scheme CUBISTA, $S_{\tau}^{\text {HOS }}$. In Eq. (9), $V$ is the volume of a cell and the factors 1.5, 2.0 and 0.5 arise from application of the three time level scheme to represent the unsteady term $\partial \tau / \partial t$ (different levels of time are denoted by superscript $(n)$ and $(*)$ denotes existing, or previous iteration, values).

The discretised momentum equation is:

$$
\begin{aligned}
a_{P} \boldsymbol{u}_{P}^{* *}= & \sum_{F} a_{F} \boldsymbol{u}_{F}^{* *}+\left\{-\nabla p^{*}+S_{u}\left[\nabla \tau^{(n+1)}\right]+S_{u}^{\mathrm{HOS}}\right. \\
& \left.+\frac{\rho V}{\delta t}\left(2.0 \boldsymbol{u}_{P}^{(n)}-0.5 \boldsymbol{u}_{P}^{(n-1)}\right)\right\}
\end{aligned}
$$

with $a_{P}=\left(1.5 \frac{\rho V}{\delta t}+\sum_{F} a_{F}\right)$ and where the coefficients $a_{F}$ have now both convective and diffusive contributions. In general, the velocity field $\boldsymbol{u}^{* *}$ obtained from implicit solution of Eq. (10) will not satisfy the discretised continuity equation:

$\nabla \cdot \boldsymbol{u}^{(n+1)}=0$

and for that reason $\boldsymbol{u}^{* *}$ and the intermediate pressure $p^{*}$ need to be corrected by $\boldsymbol{u}^{\prime}=\boldsymbol{u}^{(n+1)}-\boldsymbol{u}^{* *}$ and $p^{\prime}=p^{(n+1)}-p^{*}$. The corrected velocity field is determined from a factored form of the momentum equation:

$$
\begin{aligned}
& \left(1.5 \frac{\rho V}{\delta t}\right) \boldsymbol{u}_{P}^{(n+1)}+\left(\sum_{F} a_{F}\right) \boldsymbol{u}_{P}^{* *} \\
& =\sum_{F} a_{F} \boldsymbol{u}_{F}^{* *}-\nabla p^{(n+1)}+S_{u}\left[\nabla \cdot \boldsymbol{\tau}^{(n+1)}\right]+S_{u}^{\mathrm{HOS}} \\
& +\frac{\rho V}{\delta t}\left(2.0 \boldsymbol{u}_{P}^{(n)}-0.5 \boldsymbol{u}_{P}^{(n-1)}\right)
\end{aligned}
$$

where, by comparison with Eq. (10), we see that only the inertial and the pressure gradient terms have been updated to a new time level $(n+1)$ stage. A Poisson-like pressure correction equation is derived by subtracting Eq. (10) from Eq. (12) and imposing the divergence-free constraint (11), to yield:

$a_{P}^{p} p_{P}^{\prime}=\sum_{F} a_{F}^{p} p_{F}^{\prime}+\left\{-\left(\nabla \cdot \boldsymbol{u}^{* *}\right)\right\}$

with $a_{P}^{p}=\sum_{F} a_{F}^{p}$ and $a_{F}^{p}=A_{f}^{2} /\left(1.5 \frac{\rho V}{\delta t}\right)\left(A_{f}\right.$ cell face area).

From the above we see that all linearised sets of algebraic equations to be solved can be cast into the standard form:

$a_{P} \phi_{P}=\sum_{F} a_{F} \phi_{F}+b \Leftrightarrow[A]\{\phi\}=\{b\}$ where $b$ encompasses the terms in curled brackets in Eqs. (9), (10) and (13), and with the summation for index $F$ being over the four cell neighbours of cell $P$, in a two-dimensional application, or over the six cell neighbours in three-dimensional applications (for a local structured mesh). These large systems of equations are solved with iterative solvers: the conjugate gradient method preconditioned with an incomplete LU decomposition, for the case of the symmetric $p^{\prime}$ equation; and the bi-conjugate gradient method for the other variables (velocity and stress components).

There are two levels of iteration in the algorithm. The iterations inside the solvers, termed the "inner" iterations, are pursued until the initial residuals on entering the solver decay by two orders of magnitude. The "outer" iterations, inside a time step $\delta t$, arise because: (i) $S_{\tau}[\nabla \boldsymbol{u}]$ in the stress Eq. (9) depends on the velocity field, and $S_{\tau}[\nabla \cdot \tau]$ in the velocity Eq. (10) depends on the stress field; (ii) the factored momentum Eq. (12), devised to deal with the linear velocity/pressure coupling, is only approximate; (iii) explicit non-linearities are present in the convection terms of the momentum equation and the $f[\tau]$ function in the stress equation. These outer iterations are repeated through Eqs (9)-(13) until $\boldsymbol{\tau}^{(n+1)}, \boldsymbol{u}^{(n+1)}$ and $p^{(n+1)}$ do not change which, in practice, is achieved by controlling the normalized residuals of the equations ( $L_{1}$ norm) which are required to be below a tolerance of $10^{-4}$.

For time-dependent calculations, the procedure described above is repeated every time step until a prescribed final time is reached. If such elapsed time is sufficiently long, and if the flow in question turns out to be steady, the solution will stop varying in time and the steady-state solution is approached asymptotically as the computation proceeds. In practice this is an expensive way of calculating a steady flow and it is better in these cases to apply the procedure as a time-marching iterative solution method. For steady-state calculations, the outer iteration cycle is switched off and time advancement works effectively as equivalent to iteration, with the time step $\delta t$ playing the role of an under-relaxation factor. In this case, a final value of time need not be prescribed and time advancement will proceed until the residuals become smaller than a pre-specified tolerance (typically $10^{-4}$ for normalized residuals).

\section{Results}

In this paper, we consider only the problem of flow around the bounded cylinder placed symmetrically in a plane channel at negligible Reynolds number. A forthcoming publication will deal with the case of unbounded flow, extending the results presented in Ref. [2]. Except when explicitly indicated, distances will be scaled with the cylinder radius $R$, velocities with the average velocity in the two-dimensional channel $U$, pressure and stresses with $\eta_{0} U / R$, and the Deborah number is defined as $D e=\lambda U / R$, where $\lambda$ is the relaxation time. In what follows, we discuss issues related to boundary conditions, numerical accuracy and computational meshes, 
validation against experimental measurements, the prediction of the drag coefficient as a function of elasticity level and the occurrence of an unsteady flow regime.

\subsection{Numerical issues (meshes; boundary conditions; mesh refinement)}

For the bounded flow with a cylinder-to-channel ratio of $B \equiv R / H=0.5$, our initial computations were performed with mesh M45 of our previous work (Alves et al. [15]) which was a medium mesh for the UCM model. Due to shear thinning in the first-normal stress difference, the FENE-CR model imposes much less burden upon the numerical method and that mesh is perfectly adequate to resolve and capture with accuracy the features of the flow around the cylinder except for the normal stress variation along the downstream viscous wake. This is a very thin flow feature and proper resolution requires refinement along the $\theta$-direction for $\theta=0^{\circ}$ (i.e. clustering the mesh around the line $y=0, x>0$, from which $\theta$ is measured).

Some of the main characteristics of the various meshes are given in Table 1, including the total number of control volumes (or cells, NC), the number of control volumes around the surface of the cylinder NS, the number of cells placed radially from the cylinder to the channel wall NR (this is the figure given after the $M$ indication), and the minimum cell spacing normalized with the cylinder radius along the radial $(\delta r)$ and the azimuthal $(\delta s=r \delta \theta)$ directions. Mesh M30 has less cells in the radial direction compared with mesh M45, but is more refined along the wake; for this reason the qualification WR (for wake-refined) is added to this mesh designation. In this sense all meshes used in the present study are refined along the wake, except the original mesh M45 of [15], and another difference with Ref. [15] is that here we have also used meshes deployed over the full domain (denoted by F: full domain). Fig. 2 shows a detail of the mesh M60(WR)-FO, with an odd number (denoted by $\mathrm{O}$ in the mesh designation) of control volumes on the downstream side of the cylinder. The resolution provided by this mesh doubles that of M30(WR)-FO, by having twice as more number of cells along both directions, to give the finest mesh used in the present two-dimensional calculations with 265,680 degrees of freedom and 475 cells adjacent to the cylinder surface.

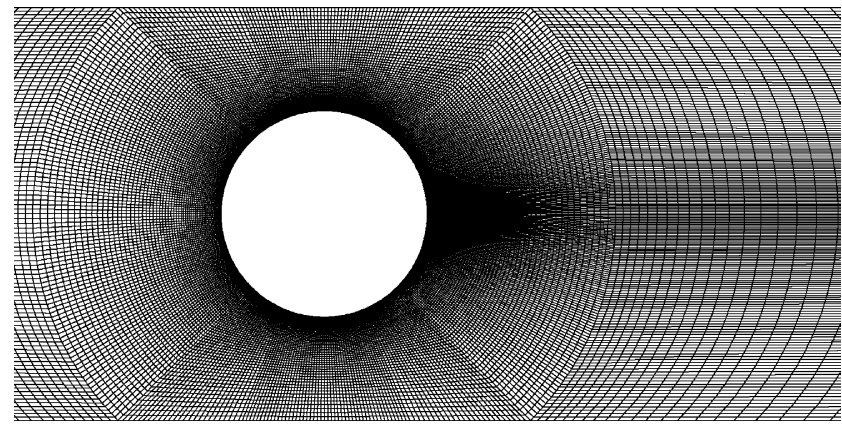

Fig. 2. Expanded view $(x \in[-3 R,+5 R], y \in[-2 R,+2 R])$ of mesh M60(WR)-FO: blockage ratio $B=0.5$, full cylinder with wake-refined mesh, odd number of cells behind cylinder.

For the half-domain meshes, symmetry is assumed about the longitudinal mid plane $(y=0)$ and therefore the boundary conditions are:

- Inlet $\left(x=-L_{1}=-20 R\right)$ : Dirichlet conditions based on analytical profiles for fully developed Poiseuille flow of the FENE-CR in a planar channel.

- Outlet $\left(x=+L_{2}=+60 R\right)$ : Neumann conditions $(\partial / \partial x \equiv 0)$ for all dependent variables, including the axial pressure gradient $\partial p / \partial x$.

- Solid walls (channel wall, $y= \pm 2 R$; cylinder surface, $r=R$ ): no slip boundary conditions for the velocity components with stresses obtained from analytical expressions.

- Symmetry plane $(y=0)$ : symmetry conditions, that is, zero normal gradients for all variables and zero normal velocity components. This boundary condition is not needed for full domain meshes.

Results for the mesh-refinement study are presented in Fig. 3, where the $u$-velocity profiles are given along the transversal direction $y$ in the narrow gap between cylinder and channel wall, and in Figs. 4 and 5, where the longitudinal variations of axial velocity $u$ and normal stress $\tau_{x x}$ components along the centerline are shown. These predictions were obtained on the two consistently refined meshes for the half-domain, M30(WR) and M60(WR) of Table 1, and for Deborah numbers of 1 and 2. It is clear that good agreement between the results from the different meshes is achieved for all quantities except the normal stress in the birefringence strand, thus indicating adequate resolution provided by the

Table 1

Some characteristics of the computational meshes

\begin{tabular}{lllrllll}
\hline Mesh & Dimension & Domain & NC & NS & $\delta r$, minimum & $\delta s$, minimum & $\delta s$, minimum wake \\
\hline M45 & 2D & Half & 9918 & 152 & 0.00646 & 0.0207 & 0.0207 \\
M30(WR) & 2D & Half & 5310 & 115 & 0.00961 & 0.0314 & 0.01 \\
M60(WR) & 2D & Half & 21240 & 230 & 0.00471 & 0.0157 & 0.005 \\
M30(WR)-F & 2D & Full & 10620 & 230 & 0.00961 & 0.0314 & 0.01 \\
M30(WR)-FO & 2D & Full/odd & 11040 & 237 & 0.00961 & 0.0314 & 0.01 \\
M60(WR)-FO & 2D & Full/odd & 44280 & 475 & 0.00471 & 0.0157 & 0.005 \\
M30 & 3D & Half & 159300 & $230 \times 30$ & 0.00961 & 0.0314 & 0.01 \\
M30 & 3D & Full & 318600 & $230 \times 30$ & 0.00961 & 0.0314 & 0.01 \\
\hline
\end{tabular}

$\mathrm{NC}$, total number of cells; NS, number of cells on cylinder surface; $\delta r, \delta s$ minimum cell spacing (normalized by $R$ ). 


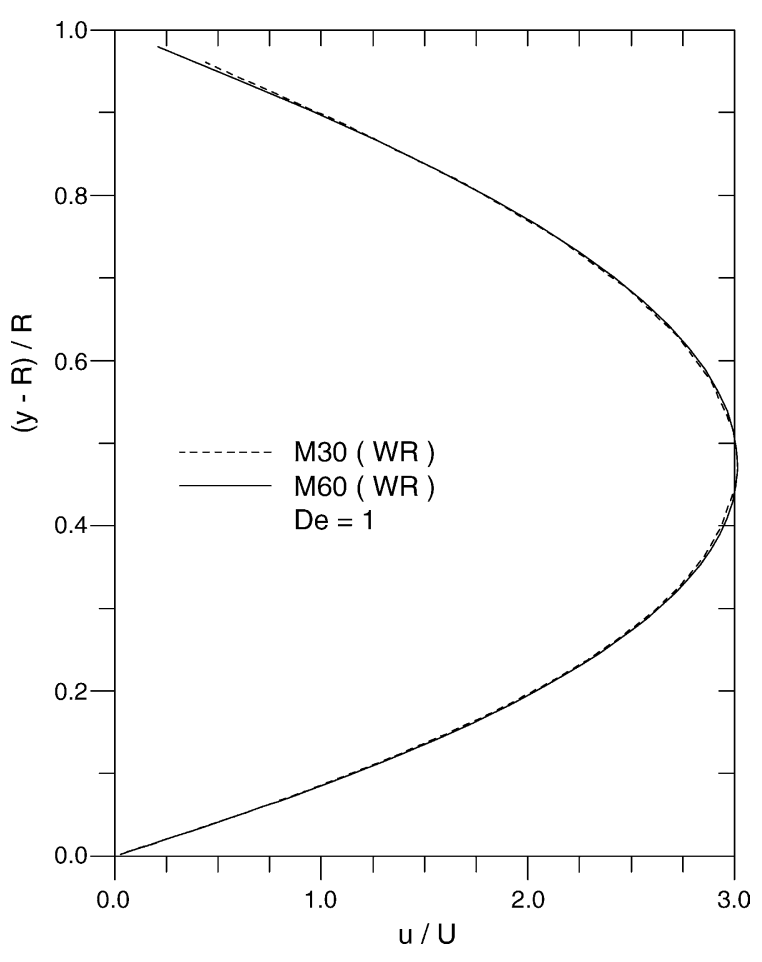

Fig. 3. Lateral profile of the $u$-velocity component in the narrow gap $(x=0)$-effect of mesh refinement for $D e=1\left(L^{2}=144\right)$.

meshes used in this study. In terms of the drag coefficient $C_{\mathrm{D}}$ obtained from integration of the full stress tensor around the cylinder surface, differences in the results obtained from the various meshes (given in Tables 2 and 3, for $L^{2}=144$ and 100, respectively) are undistinguishable in a graph and the corresponding variation with elasticity will be discussed in Section 4.3, where insensitivity to further improvement in spatial resolution will also be shown. Application of Richardson extrapolation to the $C_{\mathrm{D}}$ values obtained from the consecutively refined meshes allowed us to estimate the discretization errors: 0.08 and $0.02 \%$ on meshes M30(WR) and M60(WR), respectively, for $D e=1$ and $L^{2}=144$; and $0.03 \%$ at $D e=1$,

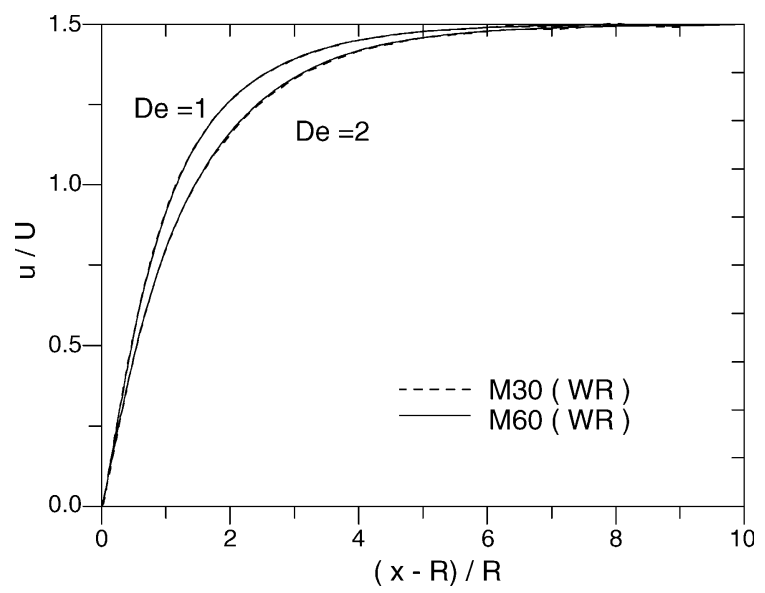

Fig. 4. Mesh refinement: velocity distribution along the cylinder wake $(y=0)$ for $D e=1$ and $2\left(L^{2}=144\right)$.

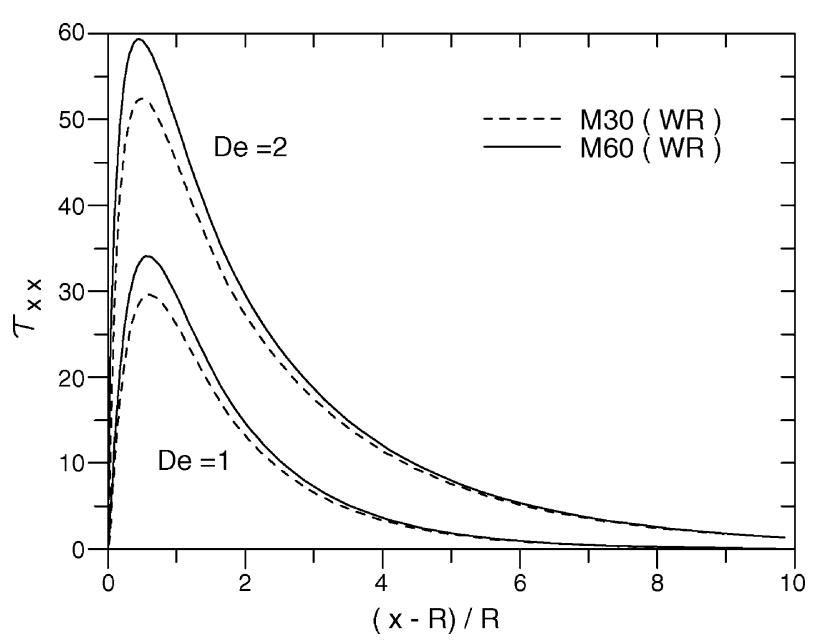

Fig. 5. Mesh refinement: normal stress distribution along the cylinder wake $(y=0)$ for $D e=1$ and $2\left(L^{2}=144\right)$.

rising to $0.24 \%$ at $D e=3.5$, for $L^{2}=100$ on mesh M30(WR). Thus, the estimated accuracy is generally better than $0.1 \%$ and only gets larger at high Deborah numbers when the flow separates and eventually becomes unsteady, as reported in Section 4.4.

The only quantity sensitive to azimuthal mesh refinement is $\tau_{x x}$ in the wake but, clearly, its exact prediction does not affect either $C_{\mathrm{D}}$ or the distribution of the other dependent variables (velocity, stress, etc.). As shown in Fig. 6 , the $\tau_{x x}$ versus $x$ variation along the line $y=0$ can be predicted more accurately by carrying out computations on a wake-refined mesh (as in [15]): the results from mesh M45 should be compared with those from M30(WR). The alternative is to employ a mesh with odd-spaced control volumes at the back of the cylinder, with a row of cells placed exactly along the "symmetry" line $y=0$. In this case, the dependent variables are calculated at positions (control volume centers) placed exactly along the downstream centreline in a full-domain mesh, without relying on interpolation, and hence, the sensitivity of the $\tau_{x x}$ prediction on mesh refinement is greatly reduced. Notice from Fig. 6 how mesh M30(WR)-FO, with just 11,040 cells, leads to almost the same predictions as mesh M60(WR) which would have $2 \times 21,240=42,480$ cells for the full domain. In addition, this figure shows that at $D e=1$ predictions of $\tau_{x x}$ on the half- and the full-domain meshes are almost undistinguishable (compare $\mathrm{M} 30(\mathrm{WR})$ and M30(WR)-F).

\subsection{Steady flow-velocity comparisons with Verhelst and Nieuwstadt (validation)}

In a recent (2004) paper Verhelst and Nieuwstadt [29] provided local velocity data obtained with LDA for the flow of both Newtonian and viscoelastic fluids around a confined cylinder with a blockage ratio of 0.5 . These data are very useful as they allow further validation of our numerical solutions, especially regarding the Newtonian flow case. For the non- 
Table 2

Drag coefficients on the various meshes with FENE-MCR for $L^{2}=144(\beta=0.59 ; R e=0)$

\begin{tabular}{|c|c|c|c|c|c|c|}
\hline$D e$ & $C_{\mathrm{D}} \mathrm{M} 45$ & $C_{\mathrm{D}} \mathrm{M} 30(\mathrm{WR})$ & $C_{\mathrm{D}} \mathrm{M} 60(\mathrm{WR})$ & $C_{\mathrm{D}} \mathrm{M} 30(\mathrm{WR})-\mathrm{FO}$ & $C_{\mathrm{D}} \mathrm{M} 60(\mathrm{WR})-\mathrm{FO}$ & $C_{\mathrm{D}}$ FENE-CR \\
\hline 0 & 132.50 & 132.44 & 132.50 & 132.44 & 132.50 & 132.50 \\
\hline 0.1 & 130.43 & 130.35 & 130.50 & 130.48 & 130.56 & \\
\hline 0.2 & 126.89 & 126.83 & 126.96 & 126.96 & 127.02 & \\
\hline 0.3 & 123.70 & 123.66 & & 123.78 & 123.82 & \\
\hline 0.4 & 121.34 & 121.33 & 121.14 & 121.44 & 121.45 & \\
\hline 0.5 & 119.77 & 119.77 & & 119.87 & 119.86 & 119.77 \\
\hline 0.6 & 118.81 & 118.84 & 118.84 & 118.90 & 118.88 & \\
\hline 0.7 & 118.29 & 118.33 & & 118.37 & 118.34 & \\
\hline 0.8 & 118.05 & 118.11 & 118.07 & 118.12 & 118.08 & \\
\hline 0.9 & 117.99 & 118.07 & & 118.05 & 117.99 & \\
\hline 1.0 & 118.04 & 118.13 & 118.03 & 118.08 & 118.01 & 118.03 \\
\hline 1.5 & 118.76 & 118.87 & 118.63 & Time-dependence & Time-dependence & 118.69 \\
\hline 2.0 & 119.35 & 119.43 & 119.13 & & & 119.23 \\
\hline 2.5 & 119.68 & 119.73 & & & & 119.55 \\
\hline 3.0 & 119.85 & 119.88 & 119.62 & & & 119.83 \\
\hline 3.5 & 119.93 & & & & & \\
\hline 4.0 & 119.97 & 119.96 & & & & \\
\hline 5.0 & 119.96 & 119.88 & & & & \\
\hline 6.0 & 119.98 & 119.76 & & & & \\
\hline 7.0 & 119.78 & 119.62 & & & & \\
\hline 8.0 & 119.66 & & & & & \\
\hline 9.0 & 119.53 & & & & & \\
\hline 10.0 & 119.39 & & & & & \\
\hline
\end{tabular}

Some FENE-CR values are also given on M45.

Newtonian fluid-flow experiments, the working fluid was a solution of polyacrylamide (150 wppm) in a Newtonian glucose $(93 \%)$ /distilled water (7\%), which was shown to be only slightly shear thinning with a zero-shear-rate viscosity ratio of $\beta=0.73$. The cylinder was mounted across a channel having a cross-sectional aspect ratio of 8 (spanwise dimension divided by channel height), a value that was not large enough to ensure a two-dimensional flow in the mid-span $x-y$ plane and so some three-dimensional effects were present, especially close to the cylinder, as stressed by the authors themselves. For the small flow rates considered the Reynolds number, although not negligible, was sufficiently low $(R e \approx 0.1-0.2)$ that inertia plays only a small role. In spite of these shortcomings, which limit the degree of expected quantitative agreement between the experiments of Verhelst and Nieuwstadt and our predictions (recall that we have $R e=0$ and a constant viscosity viscoelastic model), the comparison is still useful and, as will be seen, the important elastic effects observed in the experiments are replicated by the numerical solution.

Fig. 7 shows the comparison of the velocity profiles measured by Verhelst and Nieuwstadt [29] for the Newtonian glucose/water solution at $x=-21,-3,-1.5$ (upstream of the cylinder) and $x=+1.5,+3$, (downstream of the cylinder), with the numerical predictions for $R e=0$. The fore-aft symmetry of creeping flow is well captured by both the experimental and numerical results (coincidence for $x= \pm 3, \pm 1.5$ ), and the detailed agreement between the two is good except for the profiles closest to the cylinder $(x= \pm 1.5)$; the mi-

Table 3

Drag coefficients on the various meshes with FENE-MCR for $L^{2}=100(\beta=0.59 ; R e=0)$

\begin{tabular}{|c|c|c|c|c|}
\hline$D e$ & $C_{\mathrm{D}} \mathrm{M} 30(\mathrm{WR})$ & $C_{\mathrm{D}} \mathrm{M} 60(\mathrm{WR})$ & $C_{\mathrm{D}} \mathrm{M} 30(\mathrm{WR})-\mathrm{FO}$ & $C_{\mathrm{D}} \mathrm{M} 60(\mathrm{WR})-\mathrm{FO}$ \\
\hline 0 & 132.44 & 132.50 & 132.44 & 132.50 \\
\hline 0.1 & 130.38 & 130.54 & 130.51 & 130.60 \\
\hline 0.2 & 126.94 & 127.07 & 127.07 & 127.14 \\
\hline 0.4 & 121.60 & 121.68 & 121.71 & 121.73 \\
\hline 0.6 & 119.15 & 119.18 & 119.23 & 119.22 \\
\hline 0.8 & 118.33 & 118.32 & 118.36 & 118.34 \\
\hline 1.0 & 118.15 & 118.11 & 118.14 & 118.11 \\
\hline 1.5 & 118.34 & 118.24 & 118.24 & 118.18 \\
\hline 2.0 & 118.55 & 118.41 & 118.37 & 118.32 \\
\hline 2.5 & 118.63 & 118.51 & 118.64 & 118.37 \\
\hline 3.0 & 118.63 & 118.53 & 118.61 & 118.36 \\
\hline 3.5 & 118.60 & 118.52 & 118.53 & 118.32 \\
\hline 4.0 & 118.53 & 118.48 & Time-dependence & 118.26 \\
\hline 4.5 & 118.45 & 118.42 & & 118.19 \\
\hline 5.0 & 118.36 & 118.36 & & Time-dependence \\
\hline
\end{tabular}




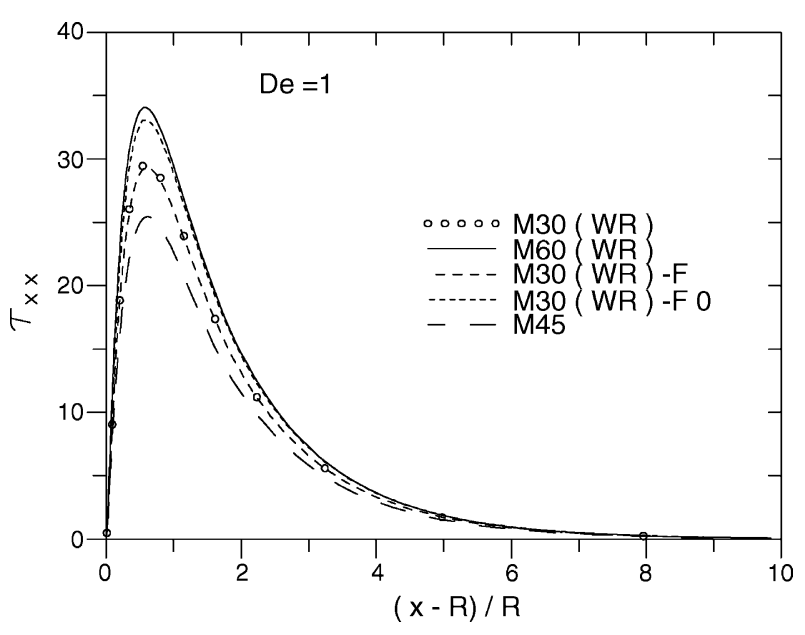

Fig. 6. Effect of mesh distribution on the normal-stress profile along the centreline $(y=0)$ for $D e=1\left(L^{2}=144\right)$.

nor differences must be attributed to three-dimensional effects because the three-dimensional numerical predictions of Verhelst and Nieuwstadt follow the data closely. We have also performed two-dimensional simulations at the Reynolds number of the experiments $\left(R e \equiv \rho U_{0} 2 R / \eta_{0}=0.23\right.$; $U_{0} \equiv$ centreline velocity) but, when plotted in dimensionless form, these predictions are indistinguishable from those for $\operatorname{Re}=0$.

So far as viscoelastic fluid flow is concerned, our numerical predictions with the FENE-MCR with $L^{2}=144, \beta=0.59$, $D e \equiv \lambda U / R=1.2$, are compared, in Fig. 8, with the measurements for a flow rate of $Q=0.020 \mathrm{l} / \mathrm{s}$, corresponding to a Deborah number of $D e=1.42$ [29]. The qualitative modifications induced by elasticity are well represented by the predictions, even without accounting for the slight shear-rate dependency

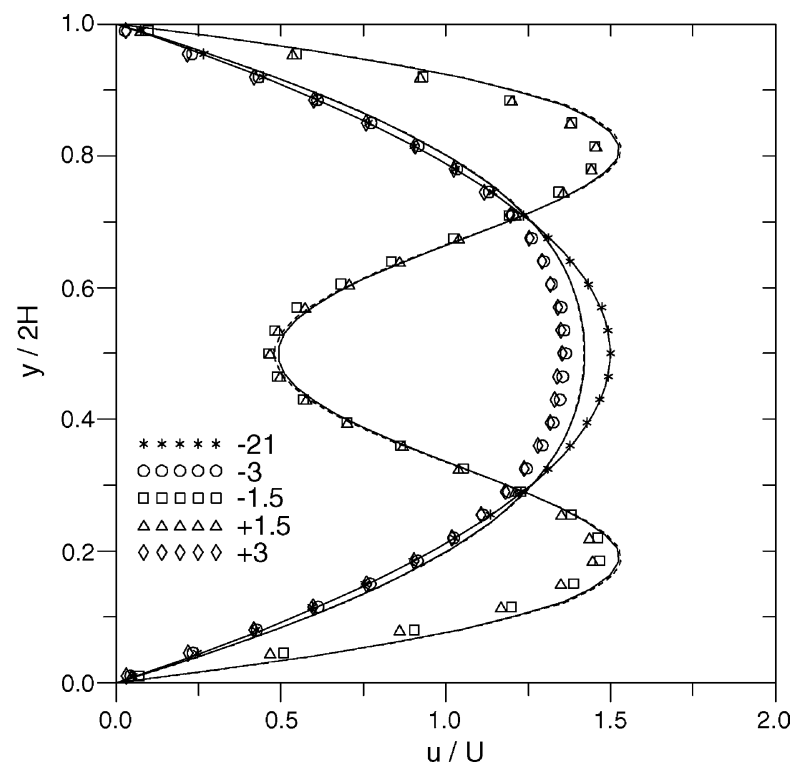

Fig. 7. Comparison of predicted velocity profiles for Newtonian fluid flow with measurements of Verhelst and Nieuwstadt [29].

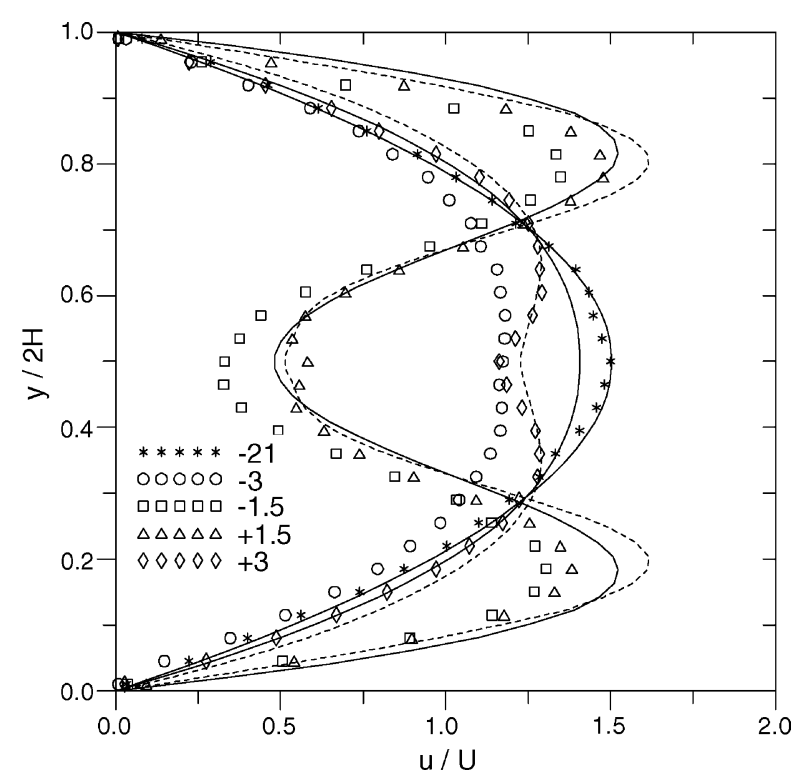

Fig. 8. Comparison of predicted velocity profiles for the FENE-MCR at $D e=1.2\left(L^{2}=144\right)$ with experimental measurements of Verhelst and Nieuwstadt [29] for a PAA solution at a flow rate of 0.0201/s $(D e=1.42)$. Dashed lines correspond to predicted downstream profiles.

of viscosity: (a) the fore-aft symmetry present in the Newtonian flow is lost due to history effects in the stress evolution: the velocity distribution at $x=-1.5$ deviated considerably from that at $x=+1.5$; (b) there is a local velocity minimum on the centreline for the velocity profiles behind the cylinder at $x=+3$, in contrast to the Newtonian fluid flow; and (c) there is also a very localised velocity maximum for the velocity profile at $x=+1.5$. This last effect is not as accentuated in these simulations as it is in the experiments because the Deborah number is lower, but the effect is visible. In fact, the influence of the birefringence strand upon the velocity field is more readily apparent in the axial velocity contours of Fig. 9, where a highly localised distortion is perceptible along the cylinder wake flow.

In conclusion, some peculiar elastic effects visible in the experimental velocity data of Verhelst and Nieuwstadt are reproduced by the present two-dimensional simulations and the Newtonian fluid-flow data are well predicted in spite of some indication of three-dimensional effects being present in the experiments. In addition, it is worth noting that Verhelst and Nieuwstadt [29] (as well as Shiang et al. [30] in previous experiments for a smaller blockage of $1 / 16$ ) do not refer to any flow instabilities, in contrast to McKinley et al. [24] and Shiang et al. [31], hence highlighting the point that such instabilities and the critical conditions for their occurrence are sensitive to the precise fluid rheology. While the viscoleastic fluid of Verhelst and Nieuwstadt [29] exhibits a slightly shear-thinning viscosity, the fluid of McKinley et al. [24] has a constant viscosity but a decreasing first normal stress coefficient with shear rate, and that of Shiang et al. [30,31] shows both constant viscosity and $N_{1}$ over the range of shear rates of interest. 


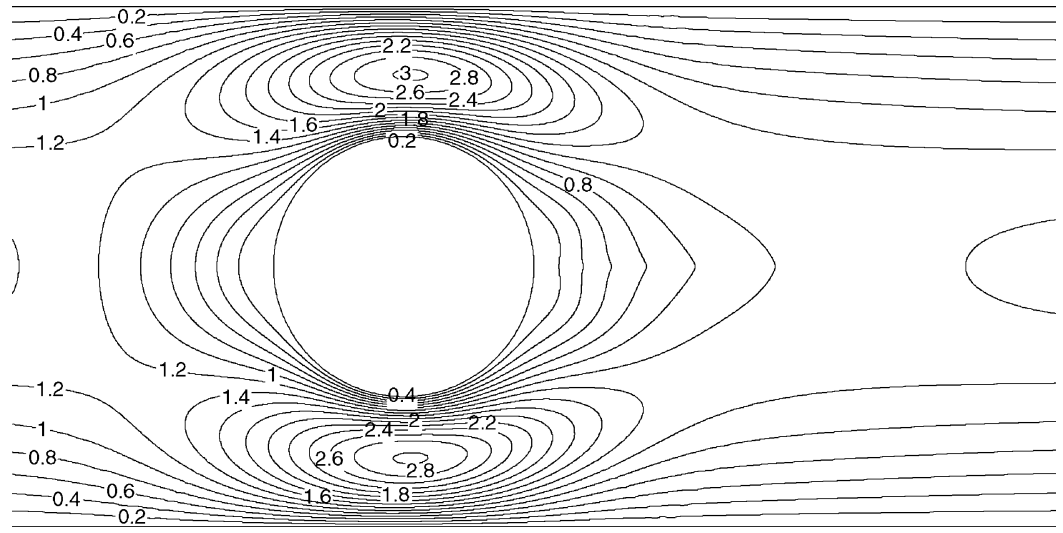

Fig. 9. Predicted contours of the axial velocity component $(u / U)$ for the FENE-MCR for $D e=1.2\left(L^{2}=144\right)$ on mesh M60(WR)-FO.

\subsection{Steady flow - drag and loss coefficients}

A convenient measure of the quality of the numerical predictions is provided by the drag coefficient obtained by integrating the total (solvent plus polymeric) stress and pressure contributions over the cylinder surface. This constitutes therefore a global solution functional, evaluated as:

$C_{\mathrm{D}}=\frac{1}{\eta_{0} U} \int_{\mathrm{cyl}}\left(-p \boldsymbol{I}+\boldsymbol{\tau}+\eta_{\mathrm{s}}\left(\nabla \boldsymbol{u}+\nabla \boldsymbol{u}^{\mathrm{T}}\right)\right) \cdot \boldsymbol{n} \cdot \hat{x} \mathrm{~d} A$

and representing the longitudinal component of the force exerted by the fluid upon the cylinder, normalized by a diffusive force scale appropriate to the inertia-free conditions under consideration. When conditions depart from creeping flow $(R e>0)$ it is more common to scale the drag force using the dynamic pressure $\left(\rho U^{2} R\right)$ when defining $C_{\mathrm{D}}$. The predicted variation of $C_{\mathrm{D}}$ with elasticity is shown in Fig. 10 for an extensibility parameter $L^{2}=144$ (see also Tables $2-4$ ), where comparison is made with the predictions for the Oldroyd-B fluid obtained by Alves et al. [15] and for the FENE-CR model at a slightly different $L^{2}=100$ by Liu et al. [10]. For $D e \geq 0.5$, the predictions of Liu et al. begin to deviate from the trend established by the current simulations, an effect that cannot be explained by the somewhat different extensibility parameter $\left(L^{2}=100\right.$, instead of 144$)$. In order to check this point we have carried out additional simulations for $L^{2}=100$ and the corresponding $C_{\mathrm{D}}$ versus $D e$ predictions, shown by a dashed lines in the figure (see data in Table 3 ), lie slightly below our predictions for $L^{2}=144$. For the Oldroyd-B fluid, Fan et al. [11] and Alves et al. [15] identified numerical divergence or unrealistic wiggles in the $\tau_{x x}$ variation behind the cylinder for $D e>1$ and therefore no steady-state solutions could be obtained for the higher Deborah number range. There was, however, a remarkable level of agreement between the $C_{\mathrm{D}}$ predictions of those authors in the range $0<D e \leq 1$ giving confidence on the correctness of their results. In addition, very recent numerical work by Kim et al. [20] with FEM and Sahin and Owens [3] with a pressure-free FVM [32] lead

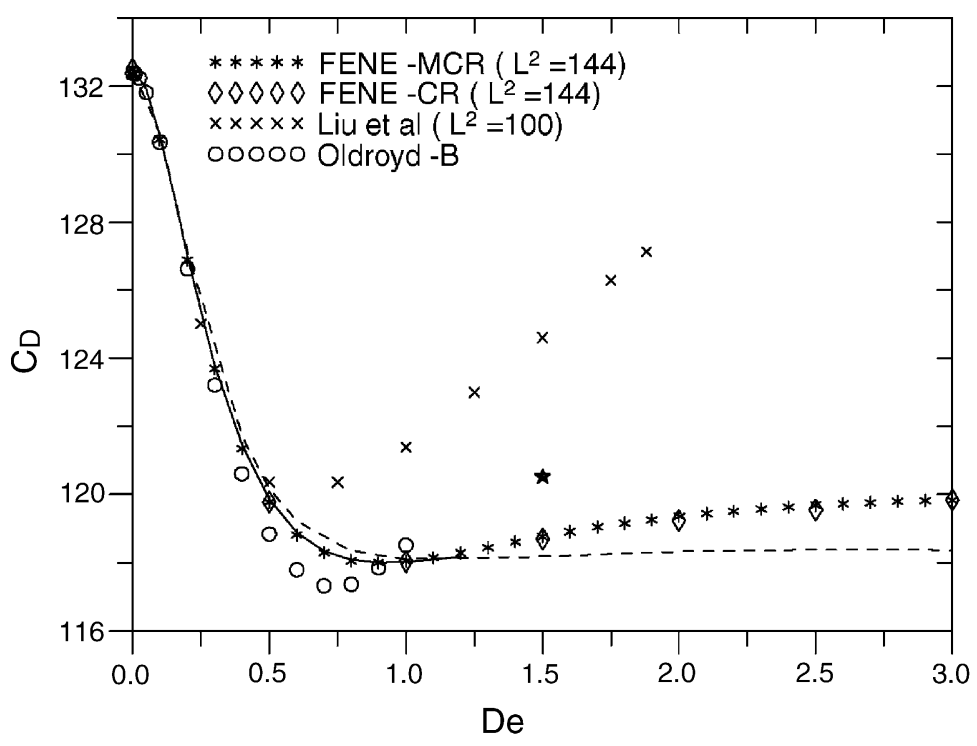

Fig. 10. Variation of the drag coefficient $C_{\mathrm{D}}$ with the Deborah number $D e$ (FENE-MCR and FENE-CR, $L^{2}=144$, mesh M45) and comparison with predictions for the Oldroyd-B [15] and FENE-CR $\left(L^{2}=100\right)$ [10] models. Additionally, predictions with the full-domain mesh M60(WR)-FO are shown with solid $\left(L^{2}=144\right)$ and dashed $\left(L^{2}=100\right)$ lines. 
to predicted $C_{\mathrm{D}}$ versus $D e$ variations which are remarkably close to the solutions of both $[11,15]$.

Fig. 10 shows a higher rate of decay of $C_{\mathrm{D}}$ with $D e$, in the range $0.5 \leq D e \leq 1.0$, for the infinite extensibility Oldroyd-B fluid compared with the FENE-MCR at $L^{2}=144$. This result suggests a stronger drag increase at higher elasticity, although results could not be obtained for these conditions either because of numerical difficulties or because the flow eventually becomes unsteady or three dimensional. This figure is also important to demonstrate that the solution obtained with the exact FENE-CR model (Eq. (4)) essentially follows the solution with the modified model (Eq. (8)), at least for the steady-state conditions under consideration.

Another possible overall flow parameter that may be useful to characterise localised losses associated with viscoelasticity, but has not been much utilised in previous studies with this flow geometry, is a loss coefficient $K$ defined as the increase in normalized pressure drop due to the presence of the cylinder. That is:

$K=\frac{\Delta p-(\Delta p)_{0}}{\eta_{0} U}$

where $(\Delta p)_{0}$ is the pressure drop between inlet and outlet, for the same conditions, but without the cylinder. $K$ is independent of the channel lengths $L_{1}$ and $L_{2}$ (see Fig. 1) provided the flow conditions are fully developed at the inlet and outlet planes: care was taken, by choosing sufficiently large $L_{1}$ and $L_{2}$ values, to ensure that these conditions were fulfilled. Values of $K$ are given in Table 4 and the variation of $K$ with $D e$ is compared in Fig. 11 with that of the drag coefficient $C_{\mathrm{D}}$. Again, it is noted that mesh refinement results in negligible variations. In addition, it may be observed that the variations of $K$ and $C_{\mathrm{D}}$ with elasticity follow essentially the same trend but with $K$ being larger than $C_{\mathrm{D}}$. This is not unexpected since the total pressure loss $K$ comprises the losses on

Table 4

Drag and loss coefficients on mesh M60(WR)-FO with FENE-MCR $\left(L^{2}=144 ; \beta=0.59 ; R e=0\right)$

\begin{tabular}{lllll}
\hline$D e$ & $C_{\mathrm{D}}$ & $C_{\mathrm{D}, p}$ & $C_{\mathrm{D}, \mathrm{s}}$ & $K$ \\
\hline 0 & 132.50 & 92.25 & 40.25 & 44.35 \\
0.05 & 131.93 & 91.73 & 40.20 & 44.18 \\
0.1 & 130.56 & 90.50 & 40.06 & 43.80 \\
0.15 & 128.82 & 88.96 & 39.86 & 43.32 \\
0.2 & 127.02 & 87.38 & 39.64 & 42.81 \\
0.3 & 123.82 & 84.64 & 39.18 & 41.92 \\
0.4 & 121.45 & 82.72 & 38.73 & 41.27 \\
0.5 & 119.86 & 81.54 & 38.32 & 40.86 \\
0.6 & 118.88 & 80.95 & 37.93 & 40.62 \\
0.7 & 118.34 & 80.77 & 37.57 & 40.52 \\
0.8 & 118.08 & 80.84 & 37.24 & 40.49 \\
0.9 & 117.99 & 81.04 & 36.94 & 40.52 \\
1.0 & 118.01 & 81.32 & 36.69 & 40.58 \\
1.1 & 118.08 & 81.61 & 36.46 & 40.65 \\
1.2 & 118.18 & 81.91 & 36.27 & 40.73 \\
1.5 & $119.84^{\mathrm{a}}$ & & & $40.76^{\mathrm{a}}$
\end{tabular}

Pressure $C_{\mathrm{D}, p}$ and shear $C_{\mathrm{D}, \mathrm{s}}$ components of drag are also given.

a Oscillates in time.

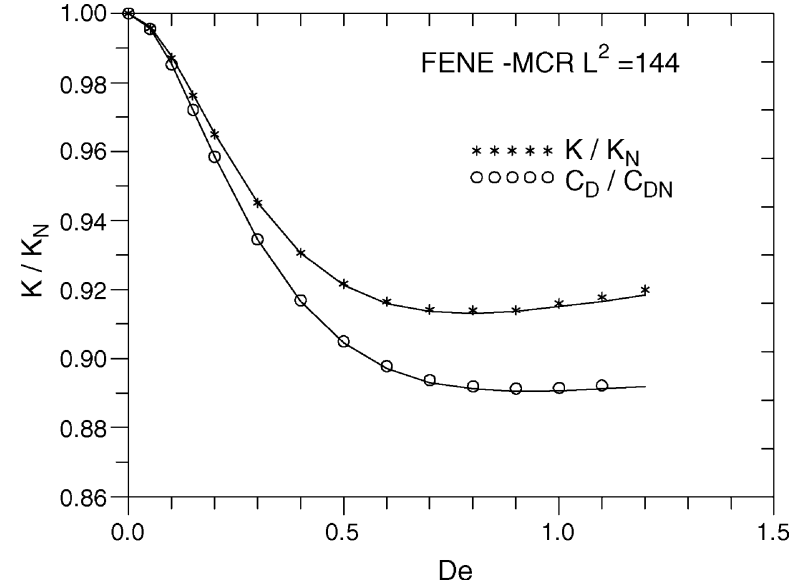

Fig. 11. Variation of the loss and drag coefficients, $K$ and $C_{\mathrm{D}}$, with Deborah number (FENE-MCR, $\left.L^{2}=144\right)$. Lines: mesh M60(WR)-FO; symbols: mesh M30(WR)-FO.

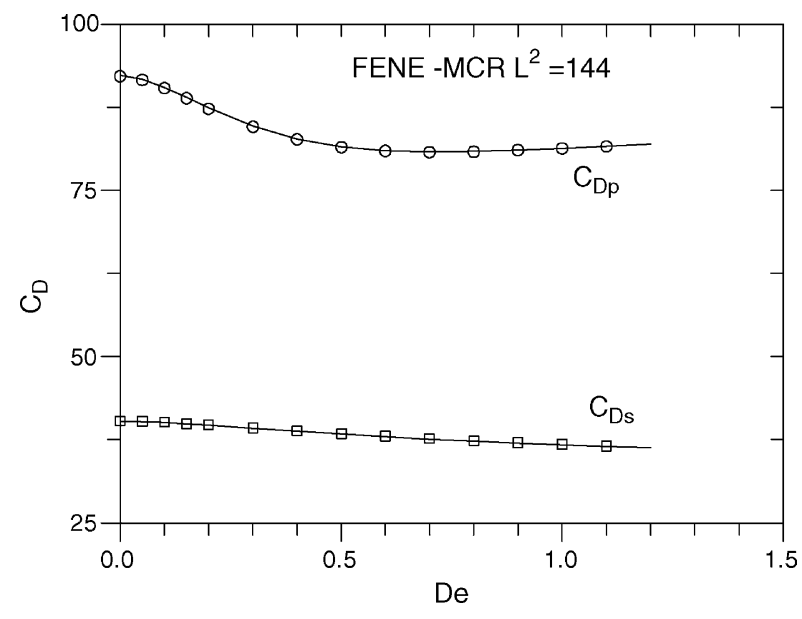

Fig. 12. Pressure and shear components of the drag coefficient (symbols: M30(WR)-FO; lines: M60(WR)-FO).

the cylinder surface, that is, $C_{\mathrm{D}}$, plus additional losses on the channel walls due to the higher shear promoted by the flow constriction in the cylinder-channel gap.

It was also possible to separate both $C_{\mathrm{D}}$ and $K$ into pressure-related (form drag) and shear-related components, and these are shown in Fig. 12 (cf. Table 4). The larger component is that due to the pressure distribution which controls the shape of the $C_{\mathrm{D}}$ variation with $D e$. In fact, the shear component remains approximately constant when $D e$ is raised.

\subsection{Unsteady flow}

The drag results of Fig. 10 were obtained with mesh M45 (or, similar results, with M30(WR)) of Table 1, which was deployed over only half of the flow domain $(y \geq 0)$, symmetry being assumed about the centre plane, $y=0$. For the full domain, the $C_{\mathrm{D}}$ values obtained from Eq. (15) need not be multiplied by a factor of 2 to account for integration over the entire cylinder surface, but otherwise no discernable differences can be seen between predictions on the 
half and full domain meshes, or indeed with the more refined meshes M60(WR) and M60(WR)-FO. However, for De > 1.3 it proved impossible to converge steady-state calculations on the full domain mesh M30(WR)-FO to arbitrarily low stopping tolerances (we recall that the time-marching method used for these calculations is iterative and some convergence criterion must be met); the residuals of the algebraic equations initially stagnate at a certain value and subsequently start fluctuating around that level. If the calculations are then pursued by tracking the solution accurately in time (with the second-order method described in Section 3), it is possible to observe that a definite time-varying regime sets in, basically confined to a small region in the near wake of the cylinder (say $1 \leq x \leq 1.2, y \leq \pm 0.2$ ), while in the rest of the domain the flow remains unvarying and steady. Since the drag coefficient is a particularly sensitive parameter to possible time-varying events occurring downstream of the cylinder (such as separation and localized recirculation), the above observations can be substantiated by tracking $C_{\mathrm{D}}$ in time, as in the plot of Fig. 13 (for $D e=1.5$ ). A perfectly sinusoidal variation of $C_{\mathrm{D}}$ versus time is observed, with a period $\approx 4.7 R / U$ (this scales with the nondimensional time for convective transport around the cylinder, $2 \pi$, divided by the nondimensional relaxation time, $D e$ ). The time-average drag coefficient for this timedependent simulation was found to be $\bar{C}_{\mathrm{D}}=120.52$, which is substantially larger than the result from steady calculations on the symmetrical domain $\left(C_{\mathrm{D}}=118.87\right.$, cf. Table 2 , shown by a "star" symbol in Fig. 10).

In order to explain the periodic variation of $C_{\mathrm{D}}$ we have considered 20 equally spaced instants in time within a period, which are marked in Fig. 13 and have been denoted by numbers from 1 to 20 , and we have observed the corresponding instantaneous streamline plots. These plots are shown in Fig. 14 for alternate instants in time, the number on top of each plot, along the main flow direction, from left to right. Twentyone streamlines are shown having equally spaced normalized stream-function values ranging from $\psi=0$ to \pm 1 (with intervals of 0.1 ) and, in addition, four other streamlines are shown

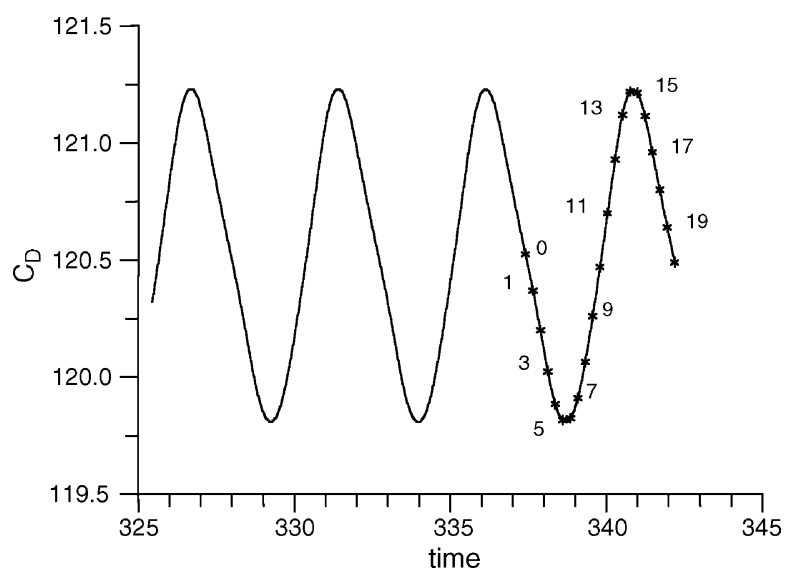

Fig. 13. Time-dependent evolution of the drag coefficient for $D e=1.5$ (FENE-MCR, $L^{2}=144$, mesh M30(WR)-FO). very close to the centerline, with $\psi= \pm 0.01$ and \pm 0.001 . The key flow feature observed in Fig. 14 is the formation of a very small recirculation zone attached to the rear of the cylinder, which changes and pulsates in time: it gradually becomes filled with the viscoelastic fluid, followed by a gradual decrease in size while the fluid partly leaves the bubble. When the bubble attains its minimum size and recirculating flow strength, at time instant five, the drag coefficient is also at a minimum; similarly, when the bubble attains its maximum size, approximately at instants $14-15, C_{\mathrm{D}}$ is at a maximum. It is noted that the bubble remains symmetric about the $x$-axis during the whole pulsating period, but the dynamic process leading to its formation can only be resolved by simulations with the full flow domain which do not rely on flow symmetry.

It is relevant to mention that the size of the time step $(\delta t)$ used in the computations is automatically adjusted in order to guarantee convergence of the iterations within a time step cycle. We started with a value of $\delta t=0.01$ (normalized with $R / U$ ), typical for this type of computation with fully implicit methods (e.g. Oliveira [6]), but as the simulation proceeded that time step was successively decreased until a value of $\delta t \cong 2.4 \times 10^{-3}$ was reached. This time step then remained constant during the computations of the periodic flow represented in Fig. 13. The streamlines of Fig. 14 are separated by a time interval corresponding to 100 such time steps.

In their experiments with a polyisobutylene $(0.31 \%)$ polymer solution in the same geometry (blockage $B=0.5$ ), McKinley et al. [24] observed a transition to a periodic timedependent flow at a shear-rate dependent Deborah number of $D e(\dot{\gamma}) \approx 1.85$, but that regime was preceded by a first transition from a steady two-dimensional to a steady threedimensional flow at a lower $\operatorname{De}(\dot{\gamma}) \approx 1.3$. This steady threedimensional flow corresponded to the formation of a cellular wake structure, with zones of high and low axial velocities in the wake repeated every characteristic wavelength along the spanwise, neutral direction (here taken as $z$ ). It is evident that three-dimensional simulations are required to capture this cellular structure but the few attempts we have taken towards that goal have been unsuccessful (these are not reported here in the interests of space, but some details of the meshes already employed are quoted in Table 1 so that the size of the problem becomes apparent). Future attempts will have to consider cyclic boundary conditions on the end planes along the $z$-direction in order to have adequate spanwise mesh resolution while still keeping the total number of control volumes (and degrees of freedom) within the limits imposed by currently available computer resources. The imposition of those boundary conditions is not without difficulties, as the wavelength of the cellular structure is not known a priori and that information influences the choice of the computational domain size along $z$. Based on the experiments of McKinley et al. we may conjecture that the time-dependent twodimensional flow resulting from the present simulations, and represented in Fig. 14, could be the triggering mechanism leading to the formation of the three-dimensional cellular structure in the wake (cf. their photograph in Fig. 14). 
(1)

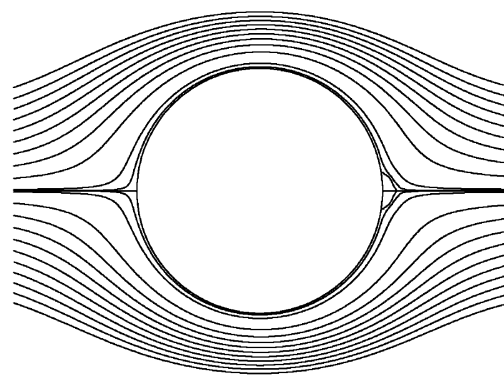

(5)

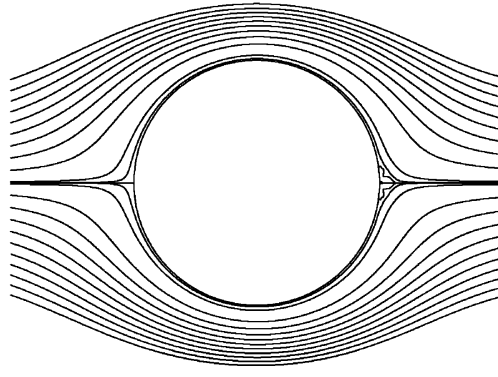

(9)

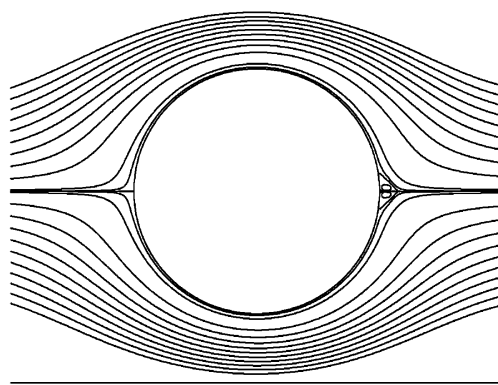

(13)

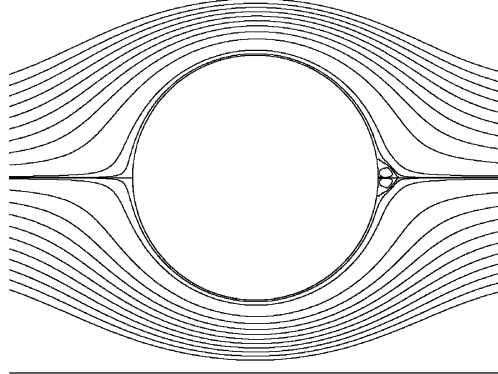

(17)

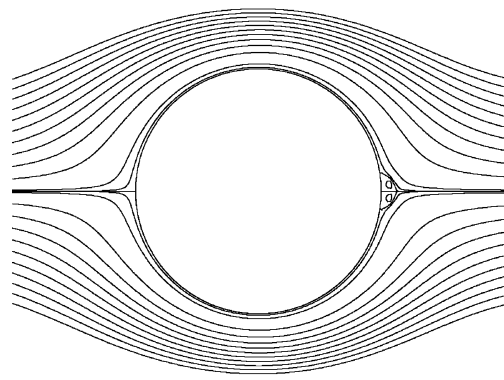

(3)
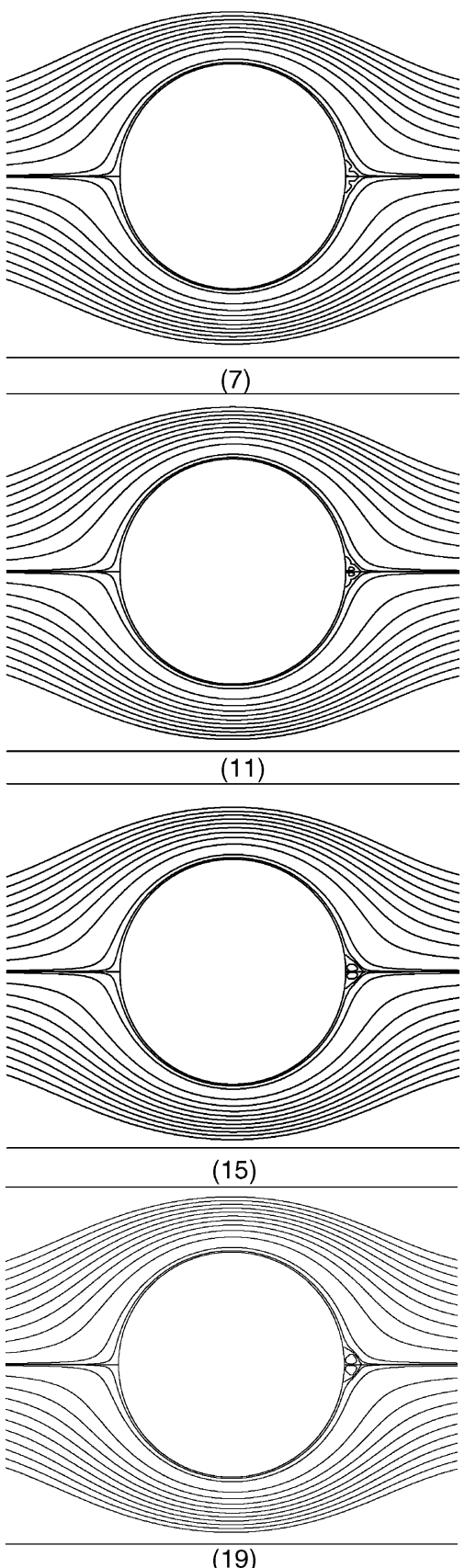

(19)

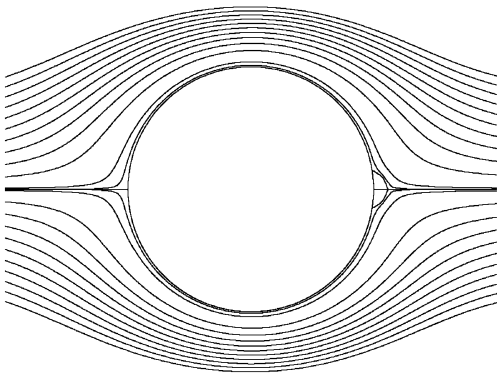

Fig. 14. Instantaneous streamline plots at equally spaced instants in time during a period, for $D e=1.5\left(L^{2}=144\right)$. 
In this respect, it is important to emphasise that the present solution shows signs of unsteadiness only in the region within the attached recirculation bubble, i.e. for $1 \leq x \leq 1.15$ and $y \leq \pm 0.15$. Outside the bubble, the flow remains basically stationary without any visible point-wise time fluctuation of the velocity and stress components. The measurements of McKinley et al. [24] closest to the cylinder are at $x=1.5$, which is outside the bubble region predicted here and also where the flow is essentially steady. On the other hand, the present unsteady flow differs from the typical vortex shedding mechanism occurring at much higher Reynolds numbers; here $R e$ is exactly equal to zero and there is no sign of an alternating shear layer mechanism. This may be illustrated by tracking streak particles released at a number of fixed points within the recirculation region and near the cylinder surface. The traces of these mass-less particles do not show any undulating motion, but essentially follow the instantaneous streamline patterns outside the recirculation (where the flow is basically steady) and leave the attached recirculation region from its furthest downstream point, at $y=0$. So far as the critical Deborah number for the first flow transition is concerned, we have found the value to be $D e \approx 1.3$, based on the zero-shear-rate relaxation time of the FENE-MCR model, while McKinley et al. [24] found the same value of $D e(\dot{\gamma}) \approx 1.3$, but with a De number based on a shear-rate dependent relaxation time which, for a FENE-CR with $L^{2}=144$ and $\beta=0.59$, corresponds to zero-shear rate Deborah number of $D e_{1}=3.8$ (equivalent to our $D e$ ). In similar experimental, work with a somewhat different PIB solution, having more elastic characteristics compared with those of McKinley's PIB fluid, Shiang et al. [31] found a critical Deborah number of $D e_{\mathrm{cr}} \approx 0.21$, but with visible effects on the flow patterns occurring only at $D e=0.66$. Their fluid had constant viscosity and constant $\psi_{1}$ for $\dot{\gamma} \leq 10 \mathrm{~s}^{-1}$, well within the typical shear rates of their experiments (it should be pointed out that local shear rates will be well above the average, calculated as $\dot{\gamma}=U / R$ ), and so their critical $D e$ is defined in a similar way as ours.

Along the previous paragraphs the extensibility parameter of the FENE-CR model was fixed at the base value of $L^{2}=144$, as suggested by [24] on account of their experimental data, and later used by [3] in their numerical calculations. It is interesting now (following the recommendation of one referee) to assess the effect on the phenomena described above of varying $L^{2}$, especially at that allows some clarification regarding the recently published work of Sahin and Owens [3], who have carried out careful time-dependent simulations on very fine meshes of flow around a cylinder with the same blockage ratio as here. To this purpose, we have considered an additional value of $L^{2}=100$, often used in connection with viscoelastic simulations with the FENECR model (see references in [2]). For this lower extensibility parameter, our predictions on both meshes M30(WR)-FO and M60(WR)-FO show that the recirculation becomes unsteady at about $D e \approx 4.0-4.5$, above which the drag coefficient and other flow features near the back of the cylinder show a periodic variation with time (albeit not exactly sinusoidal). With the mesh M30(WR)-FO a small standing eddy (without pulsation) was observed to be formed at $D e=2.5$ and unsteadiness only started for Deborah numbers above $D e \approx 3.9$. Hence, essentially the same phenomenon as described above is predicted for $L^{2}=100$ but being triggered at higher levels of elasticity. Since the simulations of Sahin and Owens [3] at their highest Deborah number, for increasing Reynolds number (see their Fig. 7), were effected at $D e=1.2$ there is no basic disagreement with the present findings, in which flow separation at the back of the cylinder is predicted to occur only at Deborah numbers above 2.5 .

\subsection{Discussion of the viscoelastic flow separation}

An explanation for the mechanism leading to numerical divergence of the simulations of steady two-dimensional viscoelastic flow around a confined cylinder at relatively low $D e$ has yet to be found, and must surely be related to the dynamic transition observed in the experiments. Based on the present predictions it seems clear that two-dimensional flow around the cylinder undergoes a bifurcation from a steady two-dimensional regime to an unsteady periodic twodimensional flow regime (preceded by flow separation and later followed by aperiodic and chaotic states) and arguably this should be related to both the difficulties in obtaining steady numerical solutions at higher $D e$, and the formation of Goertler-type three-dimensional steady regular cell-patterns along the wake of the cylinder as observed in experiments. In the light of this argument, the question that arises is why does the flow separate near the trailing edge of the cylinder, at negligible inertia $(R e=0)$ ? Most previous numerical studies ([10-16,18]) pinpoint the presence of a thin extensional viscous wake, which is also observed in experiments using the birefringence technique [33-35], and appears as a zone of high longitudinal normal stresses $\tau_{x x}$ in the downstream region of the cylinder wake. In addition, Alves et al. [15] mention the fact that the pressure behind the cylinder drops to very low values when $D e$ increases, while the MIT group (Smith et al. [14], Caola et al. [16]) prefers to explain the numerical limit as being due to the high shear stresses formed between the cylinder and the channel walls being convected into the wake.

It should be clear by inspecting the variation of $\tau_{x x}$ with $x$ behind the cylinder (cf. Fig. 5 given before) that the exceedingly high rates of growth very close to the back of the cylinder must be related to the numerical breakdown problem. With models which do not incorporate an unbounded extensional stress behaviour under simple uniaxial or planar stretching, such as the FENE-CR, the answer to the above question cannot be connected to the singular behaviour typical of UCM or Oldroyd-B viscoelastic models. Some guidelines can be obtained by inspecting the stress fields around the cylinder resulting from the numerical simulations. These are usually given as contours of the Cartesian stress components $\tau_{x x}, \tau_{y y}$ and $\tau_{x y}$ (as in $[11,12,15]$ among others). However, 
stress tensor components written in cylindrical coordinates can be advantageous due to the symmetry of the geometry in question. We have thus decided to examine the cylindrical stress components which were obtained from the basic dependent variables, the Cartesian stress components, by making use of the transformation from $(x, y)$ to $(r, \theta)$ coordinates.

In the graphs of Fig. 15 contours of these cylindrical components of the elastic extra-stress tensor (made dimensionless with $\left.\eta_{0} U / R\right)$ are given for $D e=1.2$. At this level of elasticity the flow is still entirely steady and there is at this stage no sign of recirculation downstream. Contours of the radial normal stress show the typical wake pattern already present in the $\tau_{x x}$ distribution (see, e.g. $[11,15]$ ), without showing the cylinder and channel-wall contributions characteristic of the high shear rates present in the constricted section $(x=0)$. These patterns of $\tau_{r r}$ are the typical signature of extensionalflow behaviour. The shear stress distribution, $\tau_{r \theta}$, exhibits relatively mild gradients and therefore the suggestion of the MIT group [16] does not seem to be soundly based (although it may be argued that the large azimuthal normal stresses, the effect of which is discussed below, are due to the shear flow around the cylinder). More important is the $\tau_{\theta \theta}$ stress distribution in Fig. 15b; we believe it is the fore-aft asymmetry of this stress component, which may explain the flow separation behind the cylinder with the ensuing consequences (either two-dimensional unsteady behaviour or three-dimensional

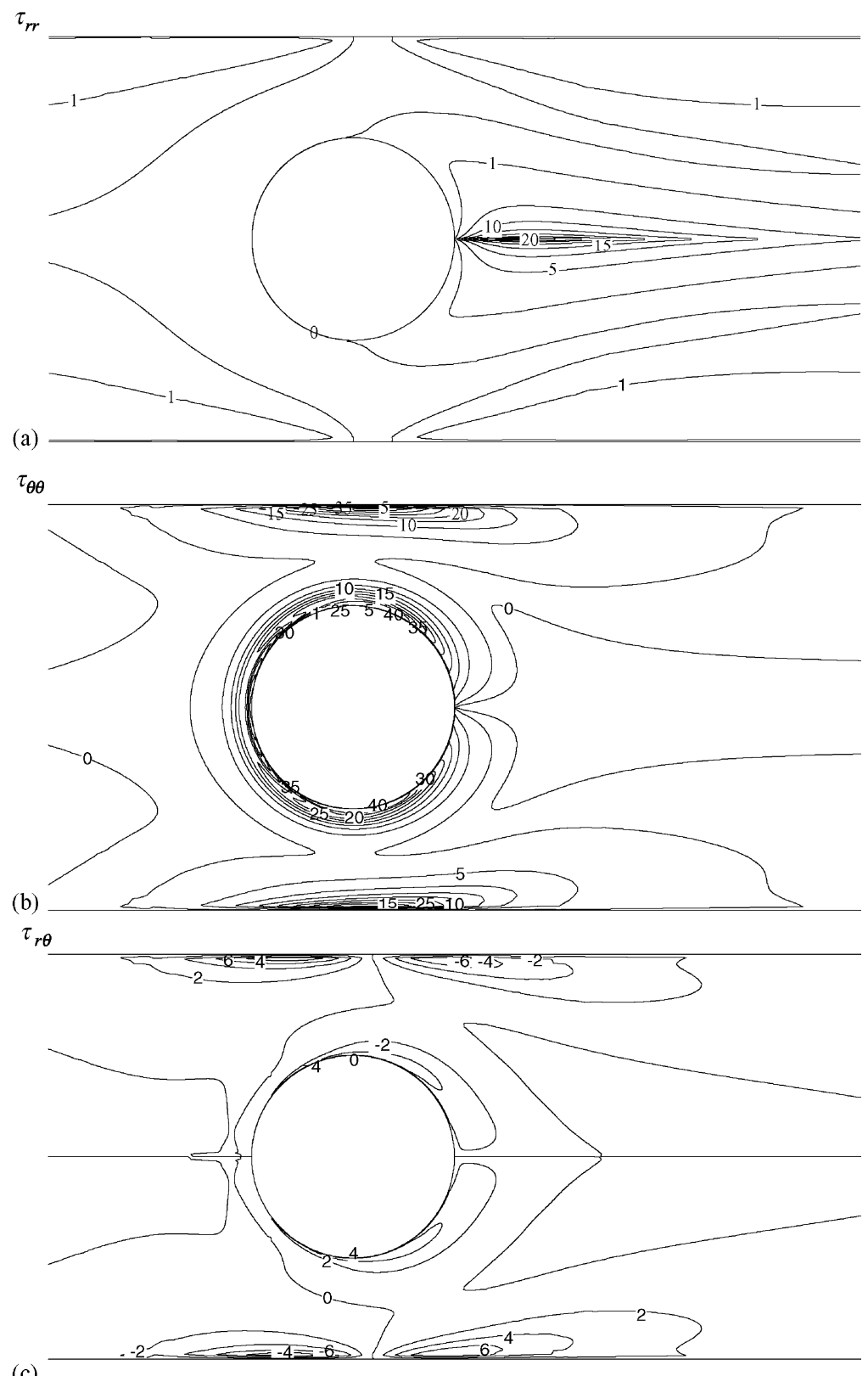

(c)

Fig. 15. Contours of the stress components in cylindrical coordinates for $D e=1.1$ on mesh M60(WR)-FO: (a) radial normal stress $\tau_{r r}$; (b) tangential normal stress $\tau_{\theta \theta}$; (c) shear-stress $\tau_{r \theta}$. 
steady cell patterns). Such asymmetry is not present for a Newtonian fluid and it is therefore a consequence of elasticity alone. Laminar-flow boundary-layer separation of a Newtonian fluid is usually explained on the basis of the influence of $\partial p / \partial x$ (where $x$ is a streamwise coordinate) at the wall: if $\partial p / \partial x<0$ the flow accelerates and no separation occurs; if $\partial p / \partial x>(\partial p / \partial x)_{\text {critic }}>0$ separation occurs (see White [36]). In the viscoelastic case in terms of cylindrical coordinates the relevant measure must be the full "streamwise" (around and along the surface of the cylinder) normal stress gradient, i.e. $\partial\left(p-\tau_{\theta \theta}\right) / r \partial \theta$.

The semi-empirical theory of Stratford explained in $\mathrm{p}$. 274 of White's book gives the critical condition for laminar separation of a Newtonian fluid in terms of the momentum thickness and it is therefore only applicable to boundary layers. At very low Reynolds numbers the flow around the confined cylinder cannot be thought of in boundary-layer terms. In order to obtain a quantitative value for the value of $(\partial p / r \partial \theta)$ at which separation occurs, we have carried out a series of simulations at increasing $R e$ and evaluated the critical condition from the numerical results. It should be noted that these were the only simulations in the present work in which the Reynolds number differed from zero. For a blockage ratio of 0.5 with a Newtonian fluid, the critical $R e$ for formation of a recirculation region behind the cylinder was found to be $R e_{\mathrm{cr}}=12.5 \pm 0.5$ and for larger Reynolds numbers the size of the attached eddies was found to increase initially (up to $R e \approx 30$ ) almost linearly with $R e$, as shown in Fig. 16, where the present predictions are compared with those of Chen et al. [37]. These authors give $R e_{\mathrm{cr}}=12.15$ in close agreement with our value, and mention that the rate of growth of the vortex size $X_{\mathrm{r}}$, as shown by the solid line in Fig. 16, is linear only in a limited range $\left(X_{\mathrm{r}} \leq 0.4\right.$, for $B=0.5)$. We have also examined the azimuthal variation of the pressure distribution around the cylinder for increasing Reynolds numbers. We find that, when the critical condition is approached, the normalized pressure gradient increases to a value of $\left(\partial p^{\prime} / r \partial \theta\right)_{\mathrm{cr}}=+1.22$, and remains almost constant

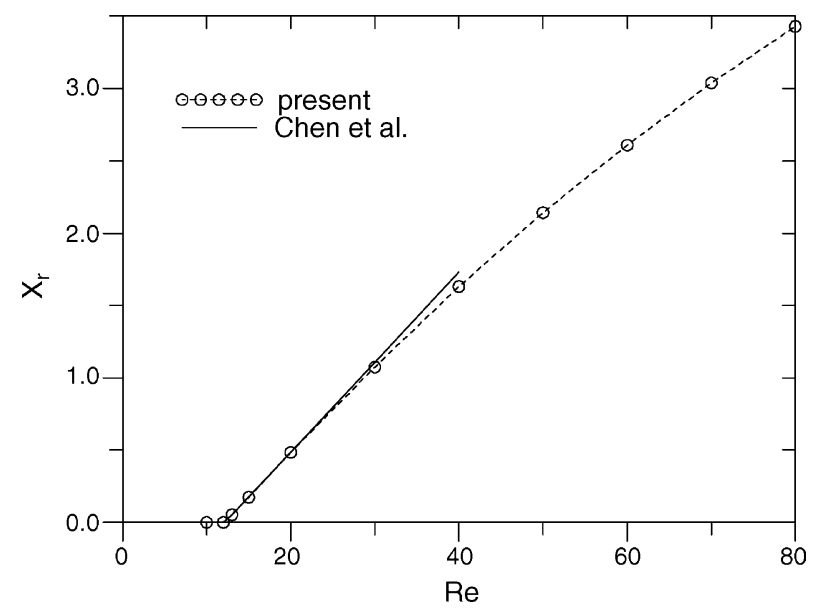

Fig. 16. Size of attached eddy as a function of Reynolds number for a Newtonian fluid, and comparison with results of Chen et al. [37]. for higher $\operatorname{Re}\left(\right.$ e.g. $\left(\partial p^{\prime} / r \partial \theta\right)_{\max } \approx+1.30$ at $\left.\operatorname{Re}=20\right)$. Here $p^{\prime}$ denotes pressure made dimensionless with $\rho U^{2}$, the appropriate scale for inertial flows, and hence the relation to our dimensionless pressure $p$ is $p=R e \times p^{\prime}$. The critical pressure gradient based on the $\eta_{0} U / R$ scale thus varies from 16 to 26 , for $R e=13$ and 20.

With the elastic fluid there is no need to increase inertia (that is, $R e$ ) to provoke flow separation. Even at zero Reynolds number conditions, a critical normal-stress (pressure plus elastic normal stress) condition similar to that found for the Newtonian case may be reached by the interplay between the pressure gradient and the elastically induced normal stress gradient. At $D e=1.2$ the pressure decays rapidly behind the cylinder, giving rise to a negative (i.e. favourable) streamwise pressure gradient, but the normal stress decays even faster and the net result is a positive $\partial\left(p-\tau_{\theta \theta}\right) / r \partial \theta \approx 24.2$. According to the Newtonian result above (which should be considered only as an estimated value) this "total" pressure gradient is on the verge of the critical condition and hence, for a slightly higher $D e$, the adverse pressure gradient cannot sustain an attached flow and separation will occur.

In summary, large positive (meaning tensile) hoop stresses $\tau_{\theta \theta}$ are generated by elastic effects as the flow passes around the cylinder. At the same time, $\tau_{\theta \theta}$ must go to zero at the "stagnation" point ( $x=R, y=0$ ) behind the cylinder (cf. Fig. 15b). It is this mechanism that induces a large negative $\partial \tau_{\theta \theta} / r \partial \theta$ which eventually leads to flow separation.

\section{Conclusions}

Computations have been performed with a finite volume method for the inertia-free (zero Reynolds number) flow of a viscoelastic FENE-MCR fluid around a cylinder confined in a planar channel with a blockage ratio of 0.5 . Steady-state results were obtained in the range $D e=0-10$ when the computational meshes covered only half of the flow domain with symmetry conditions assumed along the centerline. However, for the full domain with a set of specially designed meshes having a row of cells along the centerline in the cylinder wake, the flow was found to be unsteady and periodic for $D e>1.3$ (when $L^{2}=144$ ). A small recirculation bubble, which increases and decreases in magnitude with time in a pulsating fashion, was found to form attached to the cylinder downstream of the "stagnation" point. Due to the recirculation bubble, the drag coefficient tends to increase, compared with the steady-state situation without the bubble, and its magnitude varies sinusoidally in time at the particular value of $D e=1.5$.

With a smaller extensibility parameter of the FENE-CR model, $L^{2}=100$ instead of 144 , similar flow features were predicted but their formation was shifted to higher Deborah numbers: separation at the back of the cylinder occurred at $D e=2.5$, with a small standing eddy visible for De up to 3.9 when unsteadiness sets in.

Such a phenomenon for the flow at zero Reynolds number of non-Newtonian fluids possessing viscoelastic characteris- 
tics has not been reported in previous studies of flow around a cylinder. In some experimental investigations, however, unsteadiness behind the cylinder has been reported (see discussion in [4]) and Baaijens et al. [33] even mention the presence of measured negative axial velocities in the wake for $x \leq 1.15$.

\section{Acknowledgements}

The authors would like to record their appreciation to Dr. J. Verhelst and Prof. F. Nieuwstadt for having provided their cylinder-flow velocity data in tabulated form. The contribution of Prof. M.P. Escudier in reading and correcting a first version of the manuscript is also highly appreciated. Partial funding by Fundação para a Ciência e Tecnologia (FCT, Portugal) and FEDER under projects POCTI/EME/37711/2001 and POCTI/EME/48665/2002 is gratefully acknowledged.

\section{References}

[1] P.J. Oliveira, Asymmetric flows of viscoelastic fluids in symmetric planar expansion geometries, J. Non-Newtonian Fluid Mech. 114 (2003) 33-63.

[2] P.J. Oliveira, Method for time-dependent simulations of viscoelastic flows: vortex shedding behind cylinder, J. Non-Newtonian Fluid Mech. 101 (2001) 113-137.

[3] M. Sahin, R.G. Owens, On the effects of viscoelasticity on twodimensional vortex dynamics in the cylinder wake, J. Non-Newtonian Fluid Mech. 123 (2004) 121-139.

[4] G.H. McKinley, The nonlinear dynamics of viscoelastic flow in complex geometries, Ph.D. Thesis, MIT, Boston, MA, USA (1991).

[5] G.H. McKinley, P. Pakdel, A. Oztekin, Rheological and geometric scaling of purely elastic flow instabilities, J. Non-Newtonian Fluid Mech. 67 (1996) 19-47.

[6] P.J. Oliveira, Time-dependent simulations of shear-thinning elastic flows through contractions, ASME FED, vol. 254, Rheology and Fluid Mechanics of NonLinear Materials, 2001, p. 7 (FED24913).

[7] D.V. Boger, K. Walters, Rheological Phenomena in Focus, Elsevier, 1993.

[8] A.M. Grillet, A.C.B. Bogaerds, G.W.M. Peters, F.P.T. Baaijens, Stability analysis of constitutive equations for polymer melts in viscometric flows, J. Non-Newtonian Fluid Mech. 103 (2002) 221-250.

[9] R.A. Brown, G.H. McKinley, J. Non-Newtonian Fluid Mech. 52 (1994) 407-413.

[10] A.W. Liu, D.E. Bornside, R.C. Armstrong, R.A. Brown, Viscoelastic flow of polymer solutions around a periodic, linear array of cylinders: comparisons of predictions for microstruture and flow fields, J. NonNewtonian Fluid Mech. 77 (1998) 153-190.

[11] Y. Fan, R.I. Tanner, N. Phan-Thien, Galerkin/least-square finiteelement methods for steady viscoelastic flows, J. Non-Newtonian Fluid Mech. 84 (1999) 233-256.

[12] J. Sun, M.D. Smith, R.C. Armstrong, R.A. Brown, Finite element method for viscoelastic flows based on the discrete adaptive viscoelastic stress splitting and the discontinuous Galerkin method: DAVSS-G/DG, J. Non-Newtonian Fluid Mech. 86 (1999) 281-307.

[13] H.-S. Dou, N. Phan-Thien, The flow of an Oldroyd-B fluid past a cylinder in a channel: adaptive viscosity/vorticity (DAVSS- $\Omega$ ) formulation, J. Non-Newtonian Fluid Mech. 87 (1999) 47-73.

[14] M.D. Smith, R.C. Armstrong, R.A. Brown, R. Sureshkumar, Finite element analysis of two-dimensional viscoelastic flows to threedimensional perturbations, J. Non-Newtonian Fluid Mech. 93 (2000) 203-244.
[15] M.A. Alves, F.T. Pinho, P.J. Oliveira, The flow of viscoelastic fluids past a cylinder: finite-volume high-resolution methods, J. NonNewtonian Fluid Mech. 97 (2001) 207-232.

[16] A.E. Caola, Y.L. Joo, R.C. Armstrong, R.A. Brown, Highly parallel time integration of viscoelastic flows, J. Non-Newtonian Fluid Mech. 100 (2001) 191-216.

[17] C. Chauvière, R.G. Owens, A robust spectral element method for simulations of time-dependent viscoleastic flows, derived from the Brownian configuration field method, J. Sci. Comput. 17 (2002) 191-199.

[18] R.G. Owens, C. Chauvière, T.N. Phillips, A locally-upwinded spectral technique (LUST) for viscoelastic flows, J. Non-Newtonian Fluid Mech. 108 (2002) 49-71.

[19] X. Ma, V. Symeonidis, G.E. Karniadakis, A spectral vanishing viscosity method for stabilizing viscoelastic flows, J. Non-Newtonian Fluid Mech. (2004).

[20] J.M. Kim, C. Kim, K.H. Ahn, S.J. Lee, An efficient iterative solver and high precision solutions of the Oldroyd-B fluid past a confined cylinder, J. Non-Newtonian Fluid Mech. 123 (2004) 161-173.

[21] M.E. Mackay, D.V. Boger, An explanation of the rheological properties of Boger fluids, J. Non-Newtonian Fluid Mech. 22 (1987) 235-243.

[22] M.D. Chilcott, J.M. Rallison, Creeping flow of dilute polymer solutions past cylinders and spheres, J. Non-Newtonian Fluid Mech. 29 (1988) 381-432.

[23] P.J. Coates, R.C. Armstrong, R.A. Brown, Calculation of steady-state viscoelastic flow through axisymmetric contractions with the EEME formulation, J. Non-Newtonian Fluid Mech. 42 (1992) 141-188.

[24] G.H. McKinley, R.C. Armstrong, R.A. Brown, The wake instability in viscoelastic flow past confined circular cylinders, Philos. Trans. R. Soc. Lond. A 344 (1993) 265-304.

[25] R.B. Bird, O. Hassager, R.C. Armstrong, C.F. Curtiss, Dynamics of Polymeric Liquids, vol. 2: Kinetic Theory, Wiley, New York, 1987.

[26] R.B. Bird, J.M. Wiest, Constitutive equations for polymeric liquids, Ann. Rev. Fluid Mech. 27 (1995) 169-193.

[27] R.B. Bird, P.J. Dotson, N.L. Johnson, Polymer solution rheology based on a finitely extensible bead-spring chain model, J. NonNewtonian Fluid Mech. 7 (1980) 213-235.

[28] M.A. Alves, P.J. Oliveira, F.T. Pinho, A convergent and universally bounded interpolation scheme for the treatment of advection, Int. J. Numer. Methods Fluids 41 (2003) 47-75.

[29] J.M. Verhelst, F.T.M. Nieuwstadt, Viscoelastic flow past cylinders mounted in a channel: experimental measurements of velocity and drag, J. Non-Newtonian Fluid Mech 116 (2004) 301-328.

[30] A.H. Shiang, J.C. Lin, A. Oztekin, D. Rockwell, Viscoelastic flow around a confined cylinder: measurements using high-image-density particle image velocimetry, J. Non-Newtonian Fluid Mech. 73 (1997) $29-49$.

[31] A.H. Shiang, A. Oztekin, J.C. Lin, D. Rockwell, Hydroelastic instabilities in viscoelastic past a cylinder confined in a channel, Exp. Fluids 28 (2000) 128-142.

[32] M. Sahin, R.G. Owens, A numerical investigation of wall effects up to high blockage ratios on two-dimensional flow past a confined circular cylinder, Phys. Fluids 18 (2004) 1305-1320.

[33] F.P.T. Baaijens, H.P.W. Baaijens, G.W.M. Peters, H.E.H. Meijer, An experimental and numerical investigation of a viscoelastic flow around a cylinder, J. Rheol. 38 (1994) 351-376.

[34] W.H. Hart, D.G. Baird, The confined flow of polyethylene melts past a cylinder in a planar channel, J. Non-Newtonian Fluid Mech. 65 (1996) 247-268.

[35] F.P.T. Baaijens, S.H.A. Selen, H.P.W. Baaijens, G.W.M. Peters, H.E.H. Meijer, Viscoelastic flow past a confined cylinder of a low density polyethylene melt, J. Non-Newtonian Fluid Mech. 68 (1997) 173-203.

[36] F.M. White, Viscous Fluid Flow, second ed., McGraw Hill, 1991.

[37] J.-H. Chen, W.G. Pritchard, S.J. Tavener, Bifurcation for flow past a cylinder between parallel plates, J. Fluid Mech. 284 (1995) 23-41. 